\title{
Snail Is a Critical Mediator of Invadosome Formation and Joint Degradation in Arthritis
}

\author{
Annie Lauzier, Roxane R. Lavoie, Martine Charbonneau, Béatrice Gouin-Boisvert, Kelly Harper, and Claire M. Dubois
}

From the Immunology Division, Department of Pediatrics, Faculty of Medicine and Health Sciences, Université de Sherbrooke, Sherbrooke, Québec, Canada

\author{
Accepted for publication \\ October 7, 2015. \\ Address correspondence to \\ Claire M. Dubois, Ph.D., \\ Immunology Division, Depart- \\ ment of Pediatrics, Faculty of \\ Medicine and Health Sciences, \\ Université de Sherbrooke, 3001 \\ 12th Ave N, Sherbrooke, QC, \\ Canada J1H 5N4. E-mail: \\ claire.dubois@usherbrooke.ca.
}

\begin{abstract}
Progressive cartilage destruction, mediated by invasive fibroblast-like synoviocytes, is a central feature in the pathogenesis of rheumatoid arthritis (RA). Members of the Snail family of transcription factors are required for cell migration and invasion, but their role in joint destruction remains unknown. Herein, we demonstrate that Snail is essential for the formation of extracellular matrix-degrading invadosomal structures by synovial cells from collagen-induced arthritis (CIA) rats and RA patients. Mechanistically, Snail induces extracellular matrix degradation in synovial cells by repressing PTEN, resulting in increased phosphorylation of platelet-derived growth factor receptor and activation of the phosphatidylinositol 3-kinase/AKT pathway. Of significance, Snail is overexpressed in synovial cells and tissues of CIA rats and RA patients, whereas knockdown of Snail in CIA joints prevents cartilage invasion and joint damage. Furthermore, Snail expression is associated with an epithelial-mesenchymal transition gene signature characteristic of transglutaminase 2 /transforming growth factor- $\beta$ activation. Transforming growth factor- $\beta$ and transglutaminase 2 stimulate Snail-dependent invadosome formation in rat and human synoviocytes. Our results identify the Snail-PTEN platelet-derived growth factor receptor/phosphatidylinositol 3-kinase axis as a novel regulator of the prodestructive invadosomeforming phenotype of synovial cells. New therapies for RA target inflammation, and are only partly effective in preventing joint damage. Blocking Snail and/or its associated gene expression program may provide an additional tool to improve the efficacy of treatments to prevent joint destruction. (Am J Pathol 2016, 186: 359-374; http://dx.doi.org/10.1016/j.ajpath.2015.10.021)
\end{abstract}

Rheumatoid arthritis (RA) is a systemic autoimmune disease that mainly affects the joints, leading to rapid and disabling erosive structural damages in a proportion of patients. The onset of RA causes important morphological changes in the joint lining, including the formation of an aggressive tumorlike synovial tissue called pannus that invades and erodes the cartilage and bone. ${ }^{1}$ Pannus is composed of many cell types, including a major cell population that possesses both epithelial and mesenchymal characteristics and are referred to as fibroblast-like synoviocytes (FLS). FLS of RA patients and animal models of arthritis (A-FLS) have been shown to play a major role in invasive joint destruction through mechanisms that remain to be fully established. ${ }^{2-4}$ Arthritic FLS resemble immature and transformed mesenchymal cells, which are highly invasive in vitro and in vivo. Such property is associated with massive production of inflammatory cytokines and matrix metalloproteinases (MMPs) that sustains inflammation and joint matrix degradation. Unlike normal synoviocytes,
FLS from RA patients invade and degrade cartilage when cografted in immunocompromised mice, showing that these cells possess an imprinted invasive phenotype. ${ }^{2,5}$ Furthermore, the ex vivo invasive capability of FLS correlates with the rate of joint erosion in individual RA patients. ${ }^{6}$ Because of the predominant role played by FLS in cartilage damage, it has been proposed that an improved understanding of the mechanisms underlying their invasive and prodestructive functions may help identify strategies to stop degeneration and potentially promote regeneration of synovial joints. ${ }^{7}$

Previous work has demonstrated that arthritic FLS degrade the extracellular matrix (ECM) through formation

Supported by the Canadian Institutes for Health Research (CIHR) grant MOP-86634 (C.M.D.), membership in the Fonds de la Recherche su QuébecSanté-funded Center de Recherche du Centre Hospitalier Universitaire de Sherbrooke (C.M.D.), and a CIHR scholarship (K.H.).

Disclosures: None declared. 
of actin-rich protrusions that resemble both podosomes produced by normal cells and invadopodia produced by tumor cells. ${ }^{8,9}$ These degrading devices were called invadopodia-like structures or invadosomes. ${ }^{8,9}$ In addition to actin and the actin-binding protein cortactin, FLS invadosomes were shown to contain signaling molecules, such as activated Src and the metalloproteinases MMP-3 and MMP-13, which were all associated with cartilage damage. ${ }^{8}$ In cell cultures, invadosome production by arthritic FLS can be maintained for many weeks, further suggesting that these cells have an imprinted prodestructive phenotype. ${ }^{2}$ In collagen-induced arthritis (CIA) joints, the invadosomal structures have been detected in synovial cells strategically located at the cartilage-synovial membrane interface, for ECM invasion and degradation. ${ }^{8}$ Interference with formation of invadosomes in arthritic FLS strongly inhibited ECM degradation in vitro and ex vivo as well as FLS invasion and destruction of the underlying cartilage in an experimental model of arthritis, further linking invadosomes to joint damage. ${ }^{8,10}$ In subsequent studies, transglutaminase 2 (TG2), an enzyme important for the regulation of epithelialmesenchymal transition (EMT) and ECM composition/ stiffness, was shown to play a key role in invadosome formation by A-FLS. ${ }^{10}$ TG2 activity was associated with its ability to cross-link ECM and to up-regulate the activity and signaling of the EMT inducer, transforming growth factor (TGF)- $\beta$. Considering that the invadosome structures produced by activated FLS are functionally important to the joint degradation process, we proposed that further studies on triggers and molecular components involving invadosome biogenesis may provide cues to specifically interfere with the destructive aspect of RA. ${ }^{8}$

EMT is a transcriptionally orchestrated and multistep developmental program that is reactivated during wound healing, chronic inflammation, and neoplasias. ${ }^{11}$ During EMT, cells change their cellular phenotype to one that is more aggressive with enhanced invasiveness and resistance to apoptosis. ${ }^{12}$ Increased invasion has been associated with down-regulation of cell-cell adhesion and up-regulation of matrix metalloproteinases. ${ }^{13,14}$ These alterations are reminiscent of metastatic processes in tumors, and of cartilage invasion by pannus in arthritis. EMT can be induced by various intrinsic (eg, gene mutations) and extrinsic (eg, growth factors and matrix composition/stiffness) signals. ${ }^{12}$ In RA patients and in the CIA rat model, the synovial membrane or synovial fluid contains increased levels of EMT inducers, including the growth factors TGF- $\beta$, fibroblast growth factor, platelet-derived growth factor receptor (PDGFR), and TG2. ${ }^{8,10,15-18}$ Engagement of their cognate receptors or up-regulation of TG2 activity leads to activation of intracellular mediators, including Src, which we showed to be activated in arthritic FLS. ${ }^{8,19,20}$ All of these inducers/ pathways lead to the activation of key regulators of EMT, such as the transcription factors Snail/Slug, Twist, Zeb, and E47 that orchestrate the molecular EMT program. The molecular events include interaction with the regulatory region of epithelial or mesenchymal markers or cooperation with components of the signal transduction cascade. ${ }^{11}$ Besides their role in EMT, members of the Snail family of transcription factors, consisting of Snai1 (Snail), Snai2 (Slug), and the lesscharacterized Snail3 (Smug), have also been implicated in regulating cell proliferation, ${ }^{21}$ cell survival, ${ }^{21-24}$ and expression of stem cell properties. ${ }^{25,26}$ Moreover, Snail and Slug have been convincingly shown to be associated with a more aggressive and invasive cell phenotype. ${ }^{14,27-31}$ Recent studies have shown that Snail activity is not confined to transdifferentiating epithelial cells. Activated fibroblasts localized in damaged or cancer tissues, and macrophages in wounded and inflamed tissues, reminiscent of the microenvironment found in arthritic joints, all express increased levels of Snail, leading to cell migration and invasion. ${ }^{32-34}$ In addition, a recent study indicates that Snail is overexpressed in synovium and cell lines from RA patients. ${ }^{35}$

In light of the above findings, we hypothesized that fibroblast-like synovial cells use components or pathways of the EMT program to promote invadosome formation, leading to arthritis progression toward a more destructive phenotype. Data shown herein provide evidence that the Snail transcriptional regulator is essential for ECM degradation by human and rat synovial cells and for cartilage degradation in a CIA model. We also show that Snail mediates TG2/TGF- $\beta$-induced invadosome formation by A-FLS through down-regulation of the tumor-suppressor phosphatase and tensin homolog (PTEN) that leads to enhanced PDGFR phosphorylation and signaling. This dependency on Snail or its associated gene expression program for synovial cell-driven joint damage may be exploited to improve diagnosis and treatment of RA patients.

\section{Materials and Methods}

\section{Reagents}

LY364947 (Tocris Bioscience, Bristol, UK) and LY294002 (Cayman Chemical Co, Ann Arbor, MI) were prepared as stock solutions in dimethyl sulfoxide. AKT inhibitor XI (EMD Millipore, Billerica, MA), Bpv(pic) (Santa Cruz Biotechnology Inc., Dallas, TX), and PDGFR inhibitor V (EMD Millipore) were dissolved in water. All chemicals were purchased from Sigma-Aldrich (St. Louis, MO), unless otherwise indicated. Antibodies used were directed against cortactin (4F11; EMD Millipore), TKS5 (EMD Millipore), TG2 (TG100; Lab Vision/ NeoMarkers, Fremont, CA), tubulin (Sigma-Aldrich), Snail, PTEN, phospho-PDGFR $\alpha$ (Tyr849)/PDGFR $\beta$ (Tyr857), total PDGFR $\alpha / \beta$ (phospho-S473), and total AKT (Cell Signaling Technology Inc., Danvers, MA), and the type II collagen cleavage product Col $23 / 4 \mathrm{C}_{\text {short }}$ (Ibex Pharmaceuticals Inc., Montreal, QC, Canada).

\section{Induction and Clinical Evaluation of Arthritis}

Female Lewis/SsNHsd rats (100 to $124 \mathrm{~g}$ ) were purchased from Charles River Laboratories (Sherbrooke, QC, Canada). 
Arthritis was induced by intradermal injection of type II collagen at the base of the tail, as previously described. ${ }^{8}$ In the case of in vivo Snail (Snai1) inhibition, rats received one intra-articular injection of $3 \times 10^{9} \mathrm{U}$ of a lentivirus harboring Snail-shRNA or control (scrambled shRNA), 10 days after CIA induction. Animals were sacrificed on day 32 .

\section{Histology}

Tissue sections from human joints or the left hind knee joints of rats were processed immediately after excision, after a standardized paraffin embedding protocol. Tissue sections were rehydrated and treated with $1 \%$ trypsin (rat tissues) or $0.01 \mathrm{~mol} / \mathrm{L}$ citrate, $\mathrm{pH} 6.0$ (human tissues), and immunohistochemical staining was performed according to the standard avidin-biotin immunoperoxidase complex technique using primary antibodies (1:50 dilution in $2 \%$ bovine serum albumin) or isotype-matched negative controls and diaminobenzidene (Vector Laboratories Inc., Burlingame, CA) as substrate. For quantitative immunohistochemistry, photomicrographs of three representative areas of the synovial membrane for each joint section were captured using an Axioskop 2 phase-contrast/epifluorescence microscope (Carl Zeiss Inc., Thornwood, NY) equipped with a $10 \times$ objective and a Retiga SRV cooled color digital camera (Qimaging, Surrey, BC, Canada). Images were analyzed as previously described. ${ }^{10}$

\section{Modified Mankin Grading}

Sections were stained using hematoxylin and eosin and safranin O/fast green staining protocols to allow visualization of cartilage structure. Pathology scores were evaluated for each tissue section by three observers (A.L., M.C., and C.M.D.) in a blind way using a modified Mankin scoring system, as previously described. ${ }^{10,36}$

\section{Cell Cultures}

Rat FLS were generated using standard cell isolation and culture procedures. ${ }^{37}$ Six human cell lines isolated from patients diagnosed with RA and undergoing arthroplasty (RA-FLS) and four cell lines isolated from control joints with no evidence of disease (C-FLS) were obtained from Asterand (Detroit, MI) and Cell Applications Inc. (San Diego, CA). Cells were cultured in Dulbecco's modified Eagle's medium-F12 medium supplemented with $10 \%$ fetal bovine serum and $40 \mu \mathrm{g} / \mathrm{mL}$ gentamicin. The cells were used between passages 3 and 8 .

\section{Plasmids and Transfections}

A lentiviral construct driving the expression of green fluorescent protein (GFP)-tagged wt-TG2 was generated by subcloning the TG2 DNA fragment from pcDNA3.1 (generously provided by Dr. Gail V.W. Johnson, University of Rochester, Rochester, NY) using the following primers (5'-GGTGCTAGCGCCACCATGGCCGAGG-3', forward, and $5^{\prime}$-TATGCTAGCCCGGCGGGGCCAATGA- $3^{\prime}$, reverse) and NheI digestion, into the pLenti-V5/ GFPtag plasmid. pTK-Snail was obtained from Bob Weinberg (addgene plasmid 36976) ${ }^{38}$ and pGL3-wtPTEN from Joshua Mendell (addgene plasmid 21326). ${ }^{39}$ GFP-tagged pLenti-Snail was constructed by subcloning the Snail DNA fragment from pTK-Snail using the following primers (5'-TATGGATCCGCCACCATGCCGCGC-3', forward, and 5'-TATACTAGTGGGCGAGGGCCTCCG-3' ${ }^{\prime}$, reverse) and BamHI and SpeI digestion, into the pLenti-V5/GFPtag plasmid. GFP-lentiviral shRNA targeting rat TG2 and control (scrambled) shRNA plasmids were from EzBiolab (Carmel, IN). Control and rat/mouse Snail or PTEN targeting shRNA in pGIPZ plasmids was from Thermo Scientific (Montreal, QC, Canada). Viral particles were generated by transient transfection of 293T cells with the ViraPower lentiviral expression system (Invitrogen Thermo Fisher Scientific, Burlington, ON, Canada) or the psPAX2 (packaging plasmid) and pMD2G vectors (envelope plasmid). Experiments were conducted 24 hours after transient transfection using TransIT-LT1 (Mirus Bio LLC, Madison, WI) or 48 hours after lentivirus infection with $5 \mu \mathrm{g} / \mathrm{mL}$ Polybrene (EMD Millipore). pGIPZ transfected cells were selected by treatment with puromycin for 72 hours. pTK-Snail transfected cells were induced with $2 \mu \mathrm{g} / \mathrm{mL}$ doxycycline for 24 hours before use.

\section{Invadosome Assays}

Coverslips were covered with Alexa Fluor 488- or Oregongreen 488-conjugated gelatin (Thermo Fisher Scientific) at a final concentration of $1 \%$, as previously described. ${ }^{8}$ Cells $(30,000)$ were seeded onto each coverslip and incubated for 24 hours (rat cells) or 40 hours (human cells). Cells were fixed with $1 \%$ paraformaldehyde; nuclei and f-actin were stained with DAPI and Texas Red phalloidin, respectively; and coverslips were mounted in Vectashield (Vector Laboratories, Burlington, ON, Canada). Cells were visualized by fluorescence microscopy. Invadosomes were identified as f-actin-enriched areas of matrix degradation (loss of green fluorescence). Three fields of 100 cells were counted per coverslip. For quantification purposes of the areas of degradation, images of fluorescent matrix were analyzed using Image Pro Plus 6.0 software (MediaCybernetics, Rockville, MD), and degradation areas associated with each cell were calculated in pixels, for 10 to 15 cells per coverslip. To quantitate the number of invadosomes formed by cells, clusters of cortactin/actin were calculated for 10 to 15 cells per slide. In selected experiments, inhibitors or vehicle (control) was added 30 minutes after cell plating. When required, TGF- $\beta$ or PDGF-BB (Peprotech, Rocky Hill, NJ) was added 30 minutes after addition of the inhibitors. To identify TG2-overexpressing cells, the cells were permeabilized with $0.1 \%$ Triton X-100 for 10 minutes at room temperature. Cells were washed and blocked with $2 \%$ bovine 
serum albumin for 30 minutes, and transfected cells were examined by immunofluorescence staining using antibodies against TG2 (1:50) for 1 hour, followed by appropriate Alexalabeled secondary antibody. Invadosomes were counted in the case of TG2-positive cells only.

\section{Immunofluorescence and Confocal Microscopy}

To analyze invadosome structure formation, synoviocytes were cultured on coverslips for 4 to 6 hours and stained, as described above, using an antibody directed against cortactin (1:75), TKS5 (1:50), and Texas Red-conjugated phalloidin (Invitrogen; 1:200 or 1:50). Confocal images of the basal membrane were acquired using a Fluoview 1000 scanning confocal microscope (Olympus, Richmond Hill, ON, Canada) coupled to an inverted microscope equipped with a $60 \times$ oil immersion objective (Olympus). Color channels were scanned sequentially to avoid overlapping signals. For quantification of Snail staining, synoviocytes were cultured on coverslips for 4 to 6 hours and stained, as stated above, using an antibody directed against Snail (1:50) and TO-PRO3 iodide or DAPI (Life Technologies, Carlsbad, CA). Sets of $12 \mathrm{Z}$-stack images were collected through the thickness of the nuclei, and a merged Z-projection (maximum intensity) of the image volume $(102.4 \times 102.4 \times 12 \mu \mathrm{m} ; 12$ stacks $)$ was reconstructed using the FluoView 10-ASW version 02.01 software (Olympus). A region of interest was generated around each nuclei, and intensity of Snail staining was calculated as follows: (intensity of fluorescence-background)/surface area $\left(\mu \mathrm{m}^{2}\right)$, in a minimum of 25 cells.

\section{Transwell Invasion Assays}

Invasion assays were performed, as described by Tolboom et al, ${ }^{6}$ native using $2.0 \mathrm{mg} / \mathrm{mL}$ type II collagen-coated transwells (Corning, New York, NY).

\section{RT-PCR and PCR Array Analysis of Gene Expression}

Cells were seeded at a density of $1 \times 10^{9}$ cells $/ \mathrm{mL}$, serum starved for 6 hours, and RNA extracted using the RNeasy mini kit (Qiagen, Toronto, ON, Canada) and in situ DNase treatment, according to the manufacturer's instructions. RNA was reverse transcribed using the QuantiTect Reverse transcription kit (Qiagen). Quantitative PCR was performed using the QuantiTect SYBR Green PCR master mix (Qiagen). Primers were as follows: rat snai1, 5' GGAGCAGGAGAAAGGCTTC- $3^{\prime}$ (forward) and $5^{\prime}$ CTCTGAAGATGCACATCCGAA-3' (reverse); rat RPLP0, $5^{\prime}$-CCTTGTCTCCAGTCTTTATCAGC- $3^{\prime}$ (forward) and $5^{\prime}$-GTCACAGTACCTGCTCAGAAC-3' (reverse); human snail, 5'-CCTTCGTCCTTCTCCTCTACTT-3' (forward) and $5^{\prime}$-TTCGAGGCTGGAGATCCTT-3' (reverse); human RPLP0, 5'-GATTACACCTTCCCACTTGC-3' (forward) and 5'-CCAAATCCCATATCCTCGTCCG-3' (reverse). Analysis of expression of 84 EMT-associated genes was performed on cDNA from three control and three A-FLS cell lines using the rat Epithelial to Mesenchymal Transition $\mathrm{RT}^{2}$ Profiler PCR Array (SABiosciences Qiagen, Toronto, ON, Canada). Results were normalized to expression of Actb, B2m, Hprt1, and Rplp1, using the manufacturer's PCR array data analysis web portal.

\section{Western Blot Analysis}

Whole cell extracts were prepared by lysis of overnight serum-starved cells $\left(0.5 \times 10^{6}\right)$ in radioimmunoprecipitation assay buffer. Proteins were immunoblotted, as previously described. ${ }^{40}$ Band intensities were analyzed using the Quantity One software version 4.6.5 (Bio-Rad Laboratories, Richmond, CA).

\section{Luciferase Assay}

Cells were transiently transfected with pGL3-PTEN or pGL3-Ctl (Promega, Madison, WI) using $1 \mathrm{mg} / \mathrm{mL}$ PEI (Polysciences, Warrington, PA). Cells were serum starved and treated overnight, as indicated later, 24 hours after transfection. Cell lysates were assayed for luciferase activity, as previously described. ${ }^{41}$

\section{Statistical Analysis}

A paired or unpaired $t$-test or one-way analysis of variance test was used to assess statistical significance. $P<0.05$ was considered significant.

\section{Study Approval}

All experimental procedures involving animals were conducted under protocols (protocol 110-12B) approved by the Ethics Committee on Animal Research of the University of Sherbrooke (Sherbrooke, QC, Canada), in accordance with regulations of the Canadian Council on Animal Care. Human synovial tissue samples were obtained from RA patients who fulfill the 2010 American College of Rheumatology/European League Against Rheumatism criteria $^{42}$ and from patients with osteoarthritis (OA). All patients provided informed consent. The experimental protocol was approved by the Center Hospitalier Universitaire de Sherbrooke Ethics Committee (protocol 07-113).

\section{Results}

Snail and Snail Inducer TGF- $\beta$ Are Overexpressed in Synoviocytes of Arthritic Joints

We used a profiler PCR array consisting of 84 EMT-associated genes to assess the expression levels of EMT gene markers in synoviocytes from control and CIA rats. Comparative analysis indicated that, among the classic transcription factors involved in the EMT process (namely, Snail and Zeb family members as 


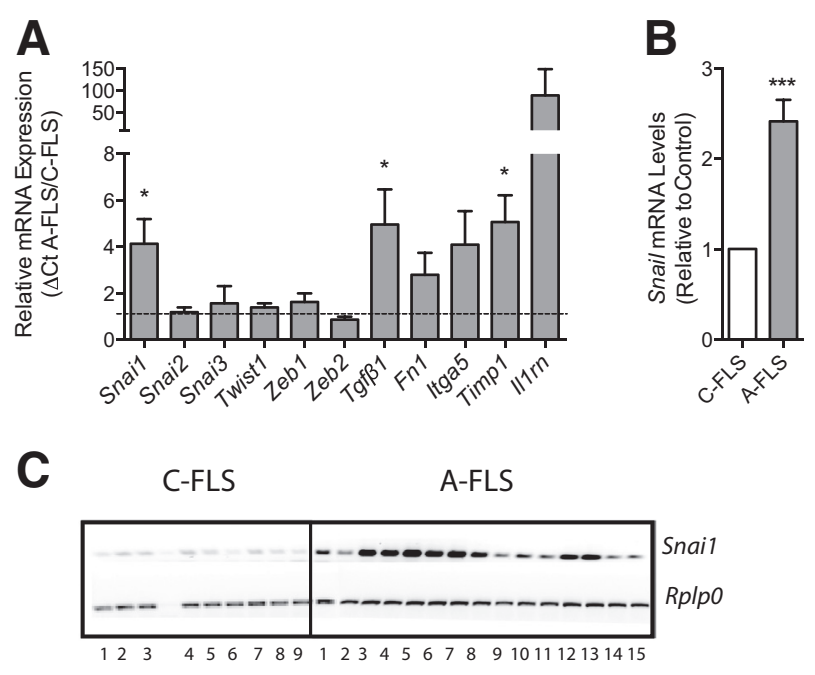

Figure 1 Snail is overexpressed in cultured fibroblast-like synoviocytes of rat collagen-induced arthritis (CIA) joints (A-FLS), control joints (C-FLS), and CIA synovial membrane. A: Quantitative RT-PCR (RT-qPCR) array results showing relative changes in the expression of epithelial-mesenchymal transition (EMT)-associated genes in A-FLS compared with C-FLS. The mean level of expression for each gene was arbitrarily set to 1 (indicated by dotted line). The $y$ axis represents fold expression, whereas the $x$ axis corresponds to genes investigated. Data normalization was calculated using the average $C_{t}$ value of the housekeeping genes Actb, B2m, Hprt1, and Rplp1. B: Total RNA from serum-starved C-FLS and A-FLS was analyzed for Snail by qPCR using Rplp0 as an internal control. Results are expressed as fold expression in A-FLS relative to C-FLS (arbitrarily set at 1). C: Representative levels of Snail1 and Rplp0 PCR amplification in individual cell lines. Data are given as means + SEM (A, analysis of variance, and $\mathbf{B}$, unpaired $t$-test). $n=3$ (A, A-FLS and $\mathrm{C}-\mathrm{FLS}) ; n=17$ (B, A-FLS and C-FLS). ${ }^{*} P<0.05, * * * P<0.001$.

well as Twist1), only Snail (encoding Snail) was significantly overexpressed, in arthritis FLS (A-FLS) compared with C-FLS (Figure 1A and Supplemental Table S1). Snail overexpression was associated with up-regulation of a sub-group of genes that comprise the Snail/EMT inducer and growth factor $T g f b l$, as well as Colla2, fn1, Itga5, Timp1, and Il1ra, which are gene targets associated with TGF- $\beta$ signaling. ${ }^{43-46}$ Validation of the data by PCR and quantitative PCR confirmed elevated Snail expression in most ( $>60 \%)$ of A-FLS compared with control cells (Figure 1, B and C). We have previously reported overexpression of TGF- $\beta$ in A-FLS. ${ }^{10}$ Snail overexpression was maintained up to eight passages of the cells (Supplemental Figure S1), which is consistent with the known capacity of arthritic FLS to maintain their destructive phenotype under in vitro conditions. ${ }^{2}$ These results indicate that arthritic FLS displayed stable overexpression of the EMT component Snail and suggest that such up-regulation is under the control of TGF$\beta$ activity.

\section{TG2/TGF- $\beta$-Induced Invadosome Formation Is Dependent on Snail}

We have shown that endogenous TGF- $\beta$ activity, triggered by the cross-linking activity of $\mathrm{TG} 2$, was essential for expression of the aggressive invadosome-forming phenotype of arthritis FLS. ${ }^{10}$ On the basis of the fact that TG2 and
TGF- $\beta$ are known inducers of EMT, we determined their implication in Snail activation in A-FLS and whether this activation was necessary for the invadosome-promoting effects of TG2 and TGF- $\beta$. We first tested the ability of TG2 to modulate Snail expression in FLS from control rats. Quantification of immunofluorescence signal intensity indicated a 2.8-fold increase in Snail expression in TG2-overexpressing cells compared with control FLS (Figure 2A). Increase in Snail expression was markedly decreased by a selective inhibitor of T $\beta R 1$ (Figure 2A). Conversely, FLS treated with TGF- $\beta$ showed a 2.0 -fold increase in Snail expression (Figure 2B). Quantitative PCR analysis of TGF- $\beta$-stimulated cell populations indicated that increased levels of Snail protein were associated with a 1.8-fold increase in Snail mRNA expression (Supplemental Figure S2). Furthermore, the expression of Snail in FLS from CIA rats returned to the levels of control cells after knockdown of endogenous TG2 or pharmacological inhibition of TGF- $\beta$ signaling (Figure 2C). The efficiency of TG2 knockdown in FLS was confirmed in a previous study. ${ }^{10}$ These observations, therefore, show that TG2 and TGF- $\beta$ increase Snail expression in FLS and that the TG2 induction of Snail is partly dependent on TGF- $\beta$ signaling. Furthermore, the data indicate that the elevated levels of endogenous Snail in arthritic FLS are under the control of the TG2-TGF- $\beta$ axis.

To determine the role of Snail in TG2- and TGF- $\beta$ induced invadosome formation, we evaluated the ability of FLS to produce invadosomes in response to TG2 overexpression or to addition of TGF- $\beta$ after transduction of Snail-specific shRNA. Snail knockdown markedly diminished the percentage of cells that produce invadosomes in response to TG2 overexpression or TGF- $\beta$ treatment (Figure 2, D-F). Furthermore, depletion of Snail in TGF$\beta$-stimulated cells resulted in a significant decrease of the gelatin degradation area (Figure $2 \mathrm{G}$ ) and the number of invadosome cores structures formed by cells identified by colocalization of actin and cortactin, two known markers of invadopodia (Figure $2 \mathrm{H}$ ). To further characterize the invadosomal structures, we stained the cells for invadosome markers actin and cortactin or actin and TKs5. Confocal microscopy of the areas of degradation formed by TGF$\beta$-treated FLS revealed cell membrane protrusions characterized by punctate actin-cortactin and actin-TKs5 cores that extended from the basal membrane of the cell into the underlying matrix, a feature typical of invadosomes (Figure 2, I and J). These results indicate that Snail is a mediator of TG2/TGF- $\beta$-induced invadosome formation and invadosome-induced ECM degradation and that upregulation of Snail could be one of the ways by which the TG2/TGF- $\beta$ axis stimulates FLS aggressiveness. ${ }^{10}$

\section{Activated Arthritis FLS Require Snail for ECM Degradation and Cartilage Invasion}

We next measured the relative levels of Snail in ECMdegrading synoviocytes from CIA rats using a combination 


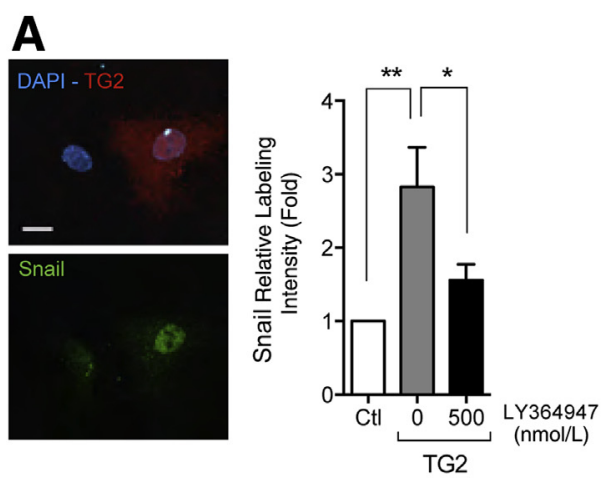

D

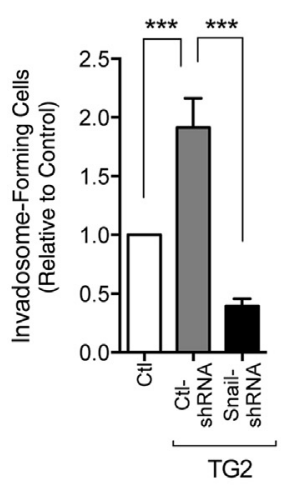

E
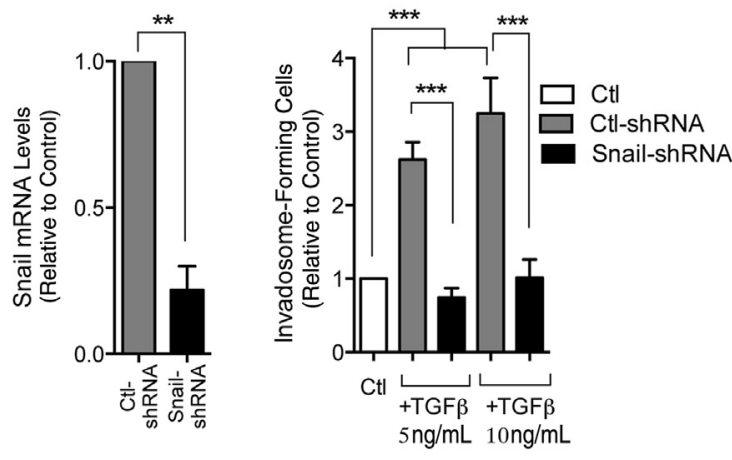

G

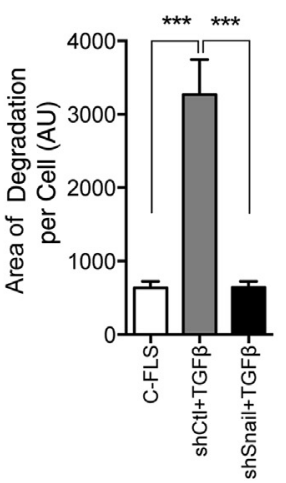

C

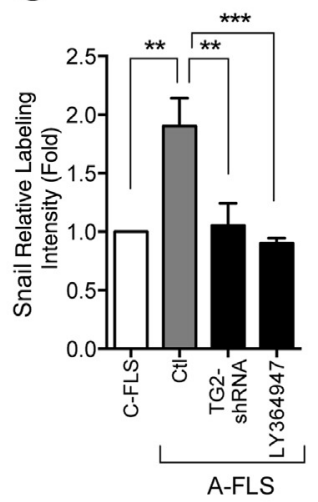

H

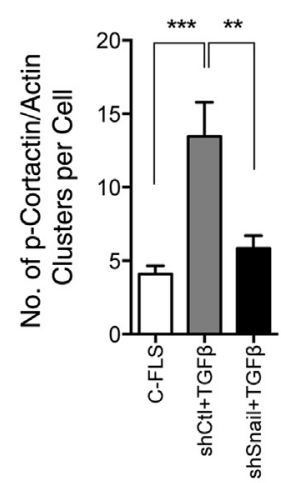

I
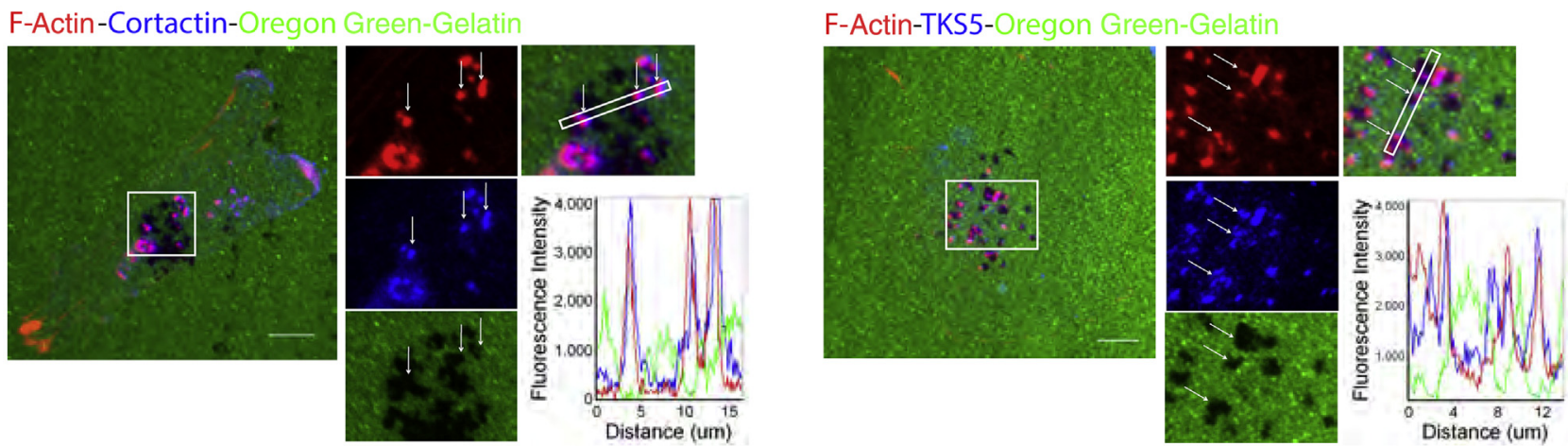

Figure 2 Snail expression is induced by transglutaminase 2 (TG2) and transforming growth factor (TGF)- $\beta$ and is essential for their invadosome-promoting activity. Control fibroblast-like synoviocytes (C-FLS) transfected with TG2 or control vector in the presence or absence of LY364947 (A) or C-FLS treated with 10 $\mathrm{ng} / \mathrm{mL}$ TGF- $\beta$ (B) were incubated for 6 hours. Representative confocal images of immunostaining for TG2, Snail (green), and nucleus (DAPI, blue) are shown. Graphs represent nuclear Snail fluorescence intensity of cells relative to C-FLS. C: C-FLS or arthritis (A)-FLS transduced with a LV encoding TG2 or scrambled [control (Ctl)] shRNA or treated with $500 \mathrm{nmol} / \mathrm{L} \mathrm{LY} 364947$ were incubated for 6 hours. Graph shows nuclear Snail fluorescence intensity of cells relative to C-FLS. Statistical differences relative to A-FLS are shown. D-J: C-FLS expressing Snail-shRNA or Ctl-shRNA were transfected with TG2 or control vector (D) or were treated with TGF- $\beta$ (F) and grown for 5 hours (confocal imaging) or 24 hours (invadosome assay) on Oregon Green 488-labeled gelatin. The percentage of cells forming invadosomes was counted for 100 transfected cells or 300 cells. Results are expressed as fold increase over control. E: Efficiency of SnailshRNA. G: Quantification of extracellular matrix degradation area per cell. H: Number of F-actin- and cortactin-positive invadosomes per cell. I and J: Representative confocal images of TGF- $\beta$-treated cells showing actin-cortactin- (I) or actin-TKS5-rich (J) invadosomes that extend into the matrix substratum. Boxed area represents higher magnification of the corresponding panel. Arrows represent areas of actin, cortactin, or TKS5 localization at areas of matrix degradation (loss of green fluorescence). The associated graphs show the fluorescence intensity profile for F-actin (red), Oregon Green 488-labeled gelatin (green), and cortactin or TKS5 (blue) through the plane indicated by the solid line of the merged high magnification image. Data represent means $+\mathrm{SEM}(\mathbf{A}-\mathbf{F}) . n=5(\mathbf{A}-\mathbf{D}) ; n=3(\mathbf{E}) ; n=4(\mathbf{F}) ; n=10$ to 15 cells $(\mathbf{G}$ and $\mathbf{H}) .{ }^{*} P<0.05, * * P<0.01$, and ${ }^{* * *} P<0.001$, paired $t$-test $(\mathbf{A}, \mathbf{B}$, and $\mathbf{D}-\mathbf{F})$ or analysis of variance $(\mathbf{C}, \mathbf{G}$, and $\mathbf{H})$. Scale bar $=10 \mu \mathrm{m}$. Original magnification, $\times 60(\mathbf{A}$ and $\mathbf{B})$. 
of invadosome assays and immunofluorescence staining. We observed that Snail was almost exclusively located in the nuclei of cells actively degrading the gelatin matrix (Figure 3A). In addition, invadosome-forming cells expressed an average of 3.2 times more Snail then degradation-inactive cells (Figure 3B), suggesting an interrelationship between the activation of Snail and ECM degradation. Furthermore, knockdown of Snail in A-FLS abolished their increased capacity to degrade ECM when compared with C-FLS (Figure 3C). These findings demonstrate that the ECM-degrading phenotype of A-FLS is dependent on Snail expression.

We next used a rat model of CIA that closely resembles RA pathology found in humans, ${ }^{47}$ to explore the role of Snail in cartilage invasion/degradation. Snail was detected by immunohistochemistry in cells of the synovial membrane and in chondrocytes of CIA tissues collected at the peak stage of the disease. There was a sixfold increase in Snail staining intensity in the synovial membrane of CIA joints relative to control synovial tissues (Figure 3D). This observation correlated with a higher number of Snail-positive cells (Figure 3D) within the synovial lining layer, further indicating that Snail is overexpressed in arthritis synovial membranes. We next examined whether silencing Snail influenced the progression of CIA. Snail-shRNA- or controlshRNA-expressing lentiviral vectors were injected intraarticularly into knee joints of rats at day 10 after arthritis induction. Hematoxylin and eosin- and toluidine-stained tissue sections from hind paws were evaluated according to a modified Mankin scoring system. ${ }^{36}$ Results showed that Snail knockdown significantly reduced Mankin score from 9.1 in tissues of CIA animals to 5.1 in tissues of SnailshRNA-treated arthritis joints (Figure 3E). Although a mild effect on synovial hyperplasia was noted (Supplemental Figure S3), most of the improvement was because of a reduction in the prevalence and depth of cartilage invasion by the synovial membrane (Figure 3F). In addition, immunostaining using an antibody directed against fragments of type II collagen showed that the extensive zones of collagen degradation observed in the joints of CIA rats were significantly reduced in tissues of CIA rats treated with SnailshRNA (Figure 3, G and H). The efficiency of Snail knockdown in synovial lining was confirmed by immunohistochemistry. Data showed that the levels of Snail detected in the synovium of Snail knockdown-treated joints were comparable to those of control tissues (Figure 3, I and J). Thus, Snail expression is associated with the aggressive invadosome-forming phenotype of arthritis FLS, and inhibition of Snail in joints efficiently reduces cartilage invasion and joint damage.

\section{Snail-Induced Extracellular Invadosome Formation Involves PTEN Repression}

Although Snail can affect many downstream pathways, few direct targets have been identified. Snail binds to the promoter region of the tumor-suppressor gene PTEN in colorectal cancer cells and represses its activity. ${ }^{24}$ Furthermore, a loss of PTEN expression has been associated with zones of aggressive cartilage invasion by RA synoviocytes. $^{48}$ To examine the effect of PTEN modulation on ECM degradation by FLS, we treated C-FLS with bpV(pic), a potent protein tyrosine phosphatase inhibitor with selectivity for PTEN. ${ }^{49,50} \mathrm{BpV}$ (pic) addition led to a dosedependent increase in invadosome-forming ability of rat FLS (Figure 4A). PTEN inhibition also caused an increase in the number of invadosomes formed per cell, as identified by clusters of actin and cortactin (Figure 4B), two bona fide markers of invadopodia core formation. ${ }^{51}$ Furthermore, enlargement of the areas of ECM degradation was observed in individual cells (Figure 4B). Similarly, knockdown of PTEN using shRNAs that targeted two independent regions of the mRNA also resulted in an increase in the number of invadosomes formed by cells and their ability to degrade the ECM (Figure 4C and Supplemental Figure S4).

We next determined whether PTEN was a transcriptional target of Snail in A-FLS. Consistent with data indicating that Snail is overexpressed in A-FLS, luciferase assays using the PTEN promoter showed a significant decrease in promoter activity in A-FLS compared with C-FLS (Figure 4D). Overexpression of Snail in control FLS resulted in a decrease of PTEN promoter activity similar to that observed in A-FLS (Figure 4D). As expected, knockdown of Snail in arthritis FLS led to an increase in PTEN expression that was similar to the level found in control FLS (Figure 4D).

Because Snail repressed PTEN promoter activity in A-FLS, we tested if PTEN repression could be a mechanism by which Snail promoted invadosome production in A-FLS. To this end, cells were transduced with a Snail-shRNA and incubated in the presence or absence of increasing concentrations of $\mathrm{bpV}$ (pic). Results showed that PTEN inhibition in A-FLS rescued the loss of invadosome formation caused by Snail depletion (Figure 4E), indicating that Snail induces matrix degradation by A-FLS through PTEN repression.

\section{Invadosome Formation in PTEN-Repressed Cells Depends on Enhanced PDGFR Activation and Signaling}

PDGFR activation is a key contributing factor in RA-FLS aggressiveness, ${ }^{52-54}$ and the phosphorylated/activated form of PDGFR might be a target of PTEN. ${ }^{55} \mathrm{We}$, therefore, examined whether PTEN inhibition promoted PDGFR activation in FLS from control rats using phosphorylationspecific antibodies in Western blot analysis. The results showed that activation-associated phosphorylation of PDGFR was strongly up-regulated in FLS treated with the PTEN inhibitor, $\mathrm{bpV}$ (pic), compared with untreated cells (Figure 4F). One major effect of the PDGFR signaling pathway involves the recruitment of phosphatidylinositol 3-kinase (PI3K) and downstream activation of AKT. ${ }^{56}$ To investigate whether the increased PDGFR phosphorylation in PTEN-inhibited cells correlated with enhanced PDGFR 
A

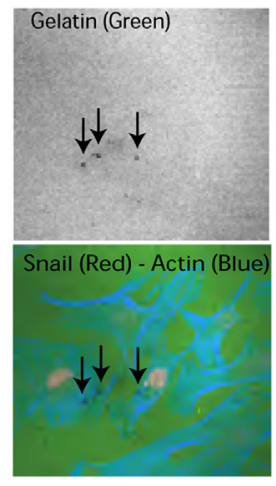

D
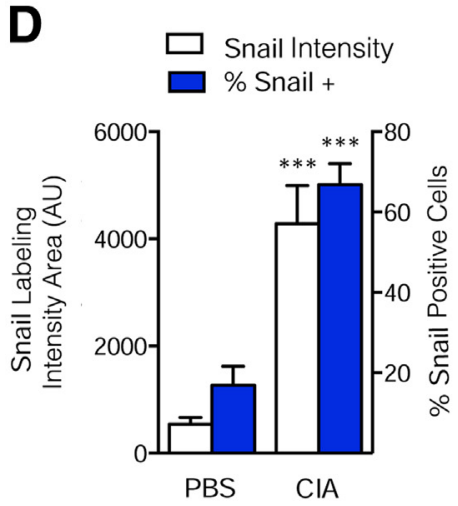

G

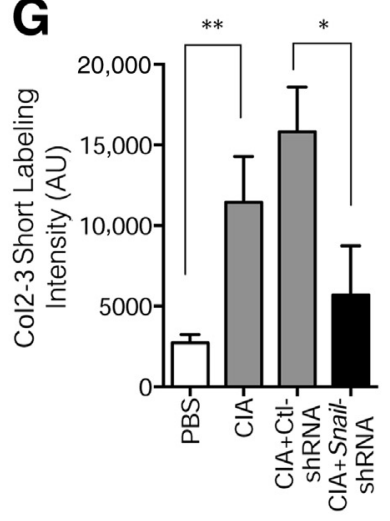

I

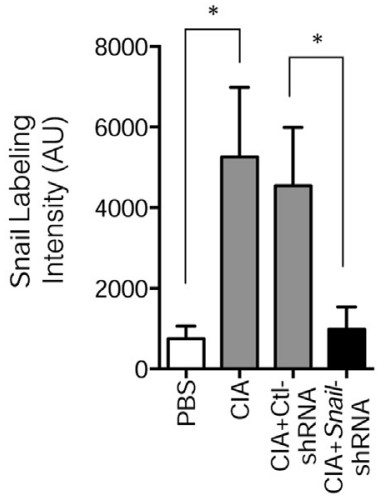

B

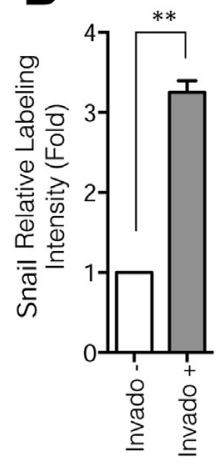

E

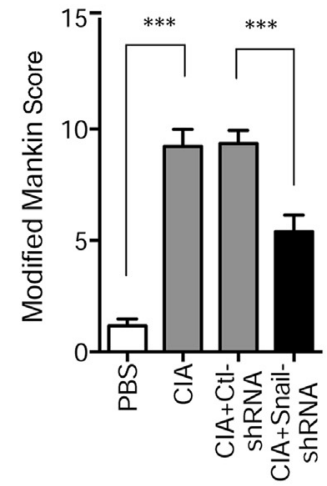

C

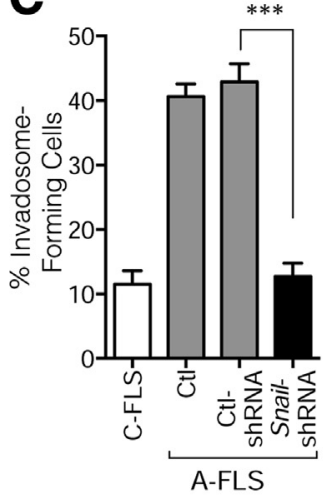

$\mathbf{F}$

$\square$ No. of Zones

$\square$ Depth

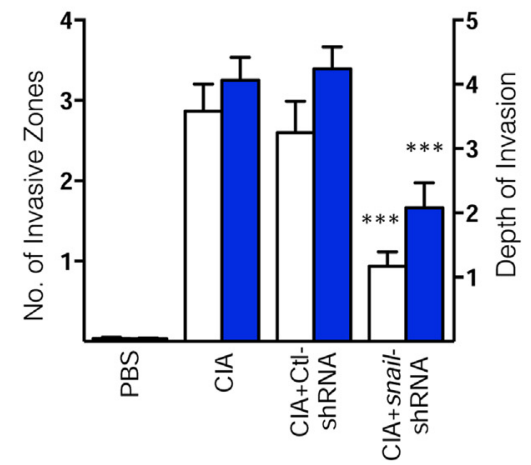

H
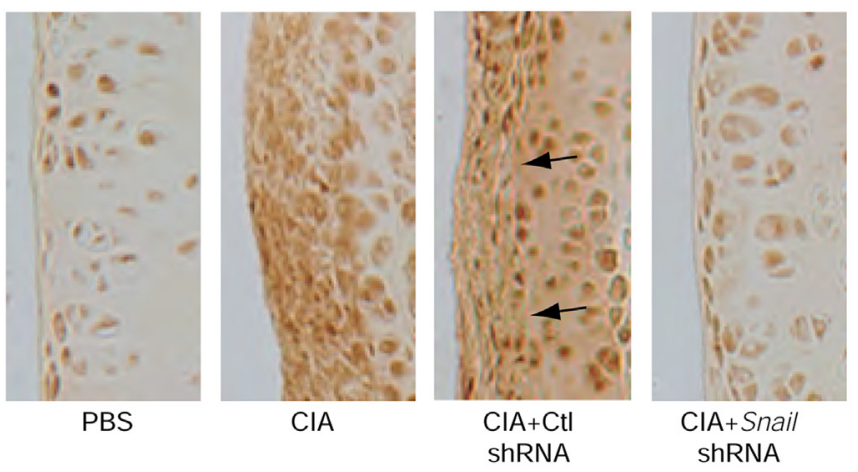

J

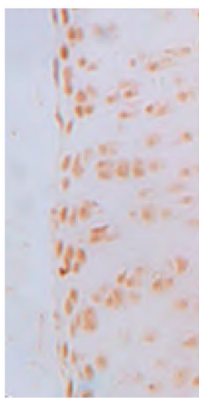

PBS

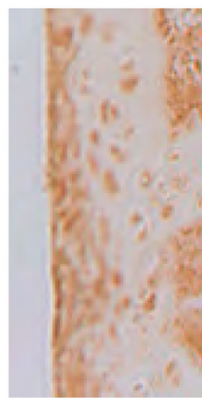

CIA

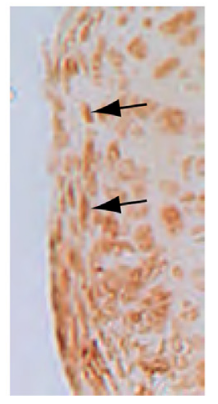

$\mathrm{ClA}+\mathrm{Ct|}$ shRNA

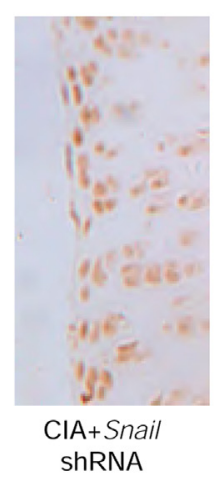


signaling, we next assessed the activation-associated phosphorylation status of AKT. Results showed that up-regulation of PDGFR activity was associated with a significant increase in AKT phosphorylation (Figure 4F), an event that was partly inhibited using a PDGFR tyrosine kinase inhibitor V, an ATP-competitive inhibitor that blocks ligand-induced PDGFR phosphorylation and kinase activity $^{57}$ (Figure 4G). These results demonstrate that PDGFR activation in PTEN-inhibited synovial cells is associated with downstream PDGFR signaling. They also suggest that part of AKT activation in PTEN-inhibited cells lies downstream of PDGFR kinase activity.

To next assess the role of the PTEN-PDGFR-PI3K/AKT axis in invadosome formation, we treated control FLS with $\mathrm{bpV}$ (pic) to remove PTEN repression, followed by the inhibition of PI3K, AKT, or PDGFR tyrosine kinase activity. Inhibition of PDGFR activity in $\mathrm{bpV}$ (pic)-treated cells resulted in strong, but partial, down-regulation of invadosome formation (Figure $4 \mathrm{H}$ ). Similar results were observed in cells treated with the PI3K inhibitor, LY 294002, and in cells treated with AKT inhibitor XI to inhibit AKT kinase activity. Overall, the data demonstrate that Snail induces the aggressive invadosome-forming phenotype of arthritic FLS through PTEN repression, resulting in the activation of downstream signaling along the PDGFR-PI3K/AKT axis.

\section{Relevance of the Snail-PTEN Axis to Human RA Cells and Tissue Synoviocytes and Synovial Tissues from RA Patients}

Our results show that Snail activated through the TG2-TGF- $\beta$ axis is an effector of invadosome formation and cartilage degradation in CIA. To explore the potential translation of these findings to human pathology, we first searched microarray databases for expression of Snail (encoded by the SNAII gene) and selected EMT markers in control (nonarthritic or OA patients) and RA synovial tissues and control and RAFLS. Comparative analysis indicated that SNAI2, TWIST, and $Z E B 2$ were significantly overexpressed in joint tissues and/or FLS of RA patients compared with control individuals (Figure 5A). In agreement with findings obtained from CIA samples, up-regulation of these EMT transcription factors was associated with a $T G F B$ gene expression signature that included increased expression of TGFBR1, TG2, COL1A2, $P D G F A$, and MMP9, and down-regulation of PTEN. ${ }^{43,46,58-60}$ Because SNAII expression was not reported in the available databases, we assessed SNAII expression in six RA synovial cell lines using RT-PCR. Results indicated that SNAII was also significantly overexpressed in RA synovial cell lines compared with control synovial cells (Figure 5B). We also examined Snail expression levels in synovial tissues. Results showed that Snail is highly expressed in the rheumatoid synovium compared with OA tissues with prominent staining in the synovial intimal lining (Figure 5C). A similar pattern of expression was observed with the phosphorylated/activated form of $S m a d 2 / 3$. Quantification of staining intensity indicated that Snail expression is significantly elevated $(P<0.001)$ in RA compared with OA (Figure 5D).

To define the role of the Snail-PTEN axis in invadosome formation by human synoviocytes, we next analyzed the ability of PTEN inhibition to reverse the loss of invadosome formation because of Snail depletion. Consistent with results obtained from rat synoviocytes, Snail was overexpressed in invadosome-forming synoviocytes from RA (Figure 6A). Knockdown of Snail inhibited invadosome formation in RA-FLS, whereas inhibition of PTEN in Snaildepleted cells rescued the ability of RA-FLS to form invadosomes (Figure 6B). Similarly, Snail inhibition strongly inhibited the ability of RA synoviocytes to invade type II collagen matrices, whereas inhibition of PTEN in control FLS promoted cell invasion (Figure 6C). These results indicated that Snail is essential for the formation of matrixdegrading invadosomes and cell invasion in human RA synovial cells and suggest that PTEN inhibition is a mechanism involved in their Snail-induced prodestructive effector function.

\section{Discussion}

\section{Synoviocytes Reactivate Snail to Gain Their Invadosome-Forming Prodestructive Phenotype}

Fibroblast-like synoviocytes are recognized as the primary effectors of cartilage degradation in RA through mechanisms that remain to be fully understood. Herein, we

\footnotetext{
Figure 3 Snail is associated with matrix degradation by fibroblast-like synoviocytes (FLS). A: Arthritis (A)-FLS grown on $1 \%$ labeled gelatin (green) for 24 hours were immunostained using an anti-Snail antibody. Representative confocal microscopy images showing Snail in A-FLS associated with areas of matrix degradation (arrows). Actin was stained with Alexa Fluor 405-labeled phalloidin (blue). B: Snail intensity for cells associated [invadosome (invado + )] or not (invado -) with areas of degradation. C: C-FLS, A-FLS, or A-FLS transduced with Snail-shRNA or (scrambled) control (Ctl)-shRNA were grown on labeled gelatin. Percentage of cells forming invadosomes (300 cells). D: Control [phosphate-buffered saline (PBS)] and collagen-induced arthritis (CIA) rat joint sections were immunostained with an antibody against Snail. Percentage of Snail-positive cells counted over the entire synovial membrane and mean Snail labeling intensity calculated in three random fields. Modified Mankin score of control (PBS), CIA, and CIA rats injected i.a. with a Snail-shRNA (CIA + Snail-shRNA) or Ctl-shRNA $($ CIA + Ctl-shRNA) expressing lentivirus 10 days after collagen or PBS injection (E) and number of zones invaded by the synovial membrane counted over the whole section and calculated mean depth of these invasion zones (F). Degraded collagen (Col2-3 short epitope) (G) and Snail labeling intensity (I) in the synovial membrane of control, CIA, and CIA rats injected i.a. with a Snail-shRNA- or Ctl-shRNA-expressing lentivirus. Representative images of degraded collagen (arrows represent degraded collagen at the synovial membrane/cartilage interface; $\mathbf{H}$ ) and Snail immunostaining (arrows represent nuclear Snail staining; J). Results are expressed as means + SEM intensity relative to cells not forming invadosomes (B) or staining intensity per area calculated from three random fields for each tissue section (G and I). $n=3(\mathbf{B}) ; n=4(\mathbf{C}) ; n=7$ (D, PBS and CIA); $n=13$ (E, PBS); $n=15$ (E, CIA and CIA + Ctl-shRNA); $n=16$ $\left(\mathbf{E}\right.$, CIA + Snail-shRNA). ${ }^{*} P<0.05,{ }^{*} P<0.01$, and ${ }^{*} * P<0.001$, paired $t$-test $(\mathbf{B})$, analysis of variance (C and $\left.\mathbf{F}\right)$, or unpaired $t$-test $(\mathbf{D}, \mathbf{E}, \mathbf{G}$, and $\mathbf{I})$.
} 
A

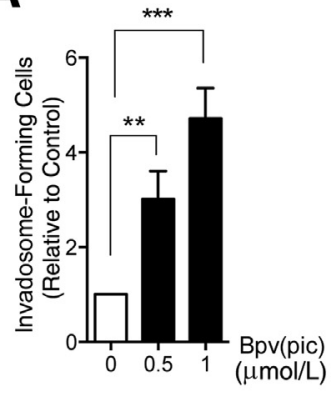

D

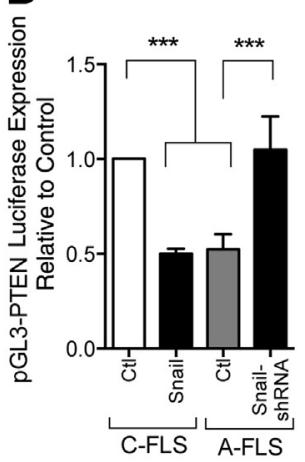

G

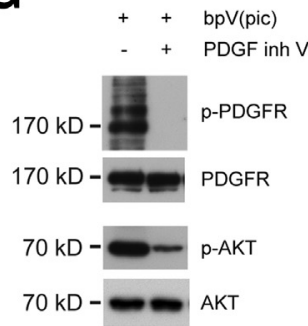

B

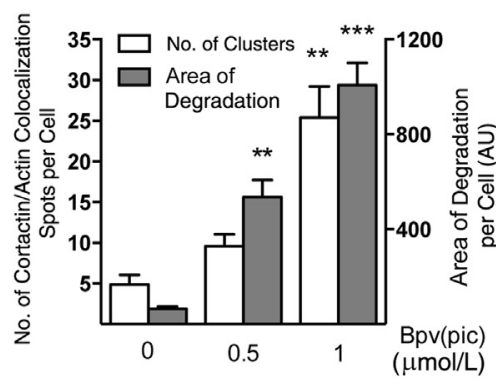

E

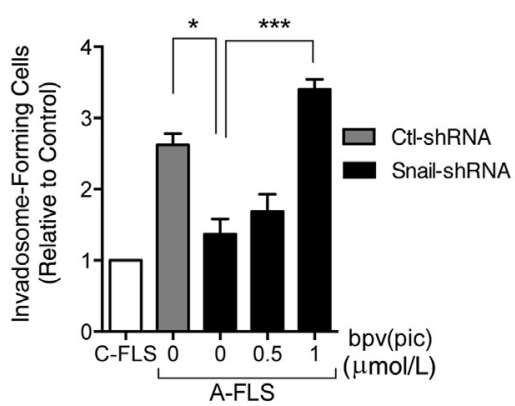

C

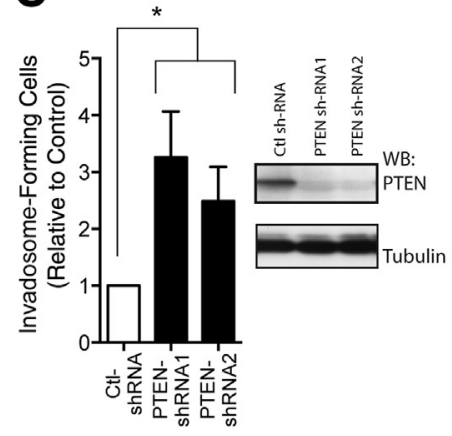

F

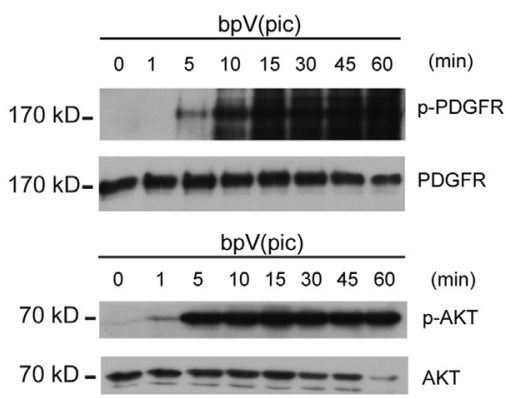

H

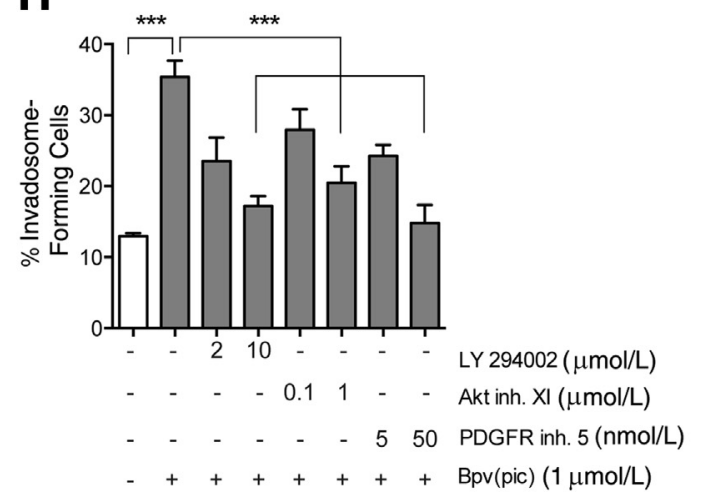

Figure 4 Snail-mediated matrix degradation involves PTEN repression. A: Control fibroblast-like synoviocytes (C-FLS) were cultured on gelatin for 24 hours with or without $\mathrm{Bpv}(\mathrm{pic})$ at the indicated concentrations. The percentage of cells forming invadosomes was calculated. Results are expressed relative to control cells treated with vehicle. B: Quantification of the number of invadosomes formed (actin/cortactin clusters) and the corresponding area of degradation per cell. C: C-FLS transduced with phosphatase and tensin homolog (PTEN)-shRNA or control (Ctl)-shRNA were evaluated in an invadosome assay. PTEN knockdown was assessed by immunoblotting. D: Luciferase reporter assay showing PTEN promoter activity in C-FLS transfected with an empty- or Snail-encoding vector and A-FLS transduced with Snail1-shRNA or Ctl-shRNA. E: Percentage of invadosome-forming cells in A-FLS transduced with Snail-shRNA or Ctl-shRNA. Results are expressed relative to control cells. $\mathbf{F}$ and $\mathbf{G}$ : Representative immunoblots of p-platelet-derived growth factor receptor (pPDGFR), PDGFR, pAKT, and AKT after incubation (1 to 60 minutes) of C-FLS with 1 of C- of bpV(pic) (F) or incubation (15 minutes) of C-FLS with 1 of C- of bpV(pic) with or without $50 \mathrm{nmol} / \mathrm{L}$ PDGFR inhibitor V (G). H: C-FLS were pretreated for 30 minutes with inhibitors before addition of $1 \mu \mathrm{mol} / \mathrm{L}$ bpV(pic) where indicated. Percentage of cells associated with invadosomes is shown. Data represent means + SEM (A-E and $\mathbf{H}) . n=5(\mathbf{A}) ; n=3(\mathbf{B}-\mathbf{E}) ; n=4(\mathbf{H}) .{ }^{*} P<0.05$, $* * P<0.01$, and ${ }^{* *} P<0.001$, analysis of variance $(\mathbf{A}, \mathbf{B}, \mathbf{C}, \mathbf{E}$ and $\mathbf{H})$ or unpaired $t$-test (D). Inh., inhibitor; WB, Western blot.

identified Snail as a critical regulator of the prodestructive invadosome-forming phenotype of FLS of rat and RA patients and cartilage degradation in experimental arthritis. Snail exerts its function through direct down-regulation of PTEN transcription, resulting in increased PDGFR phosphorylation and PI3K/AKT activation (Figure 7). Enhanced Snail gene expression was associated with a
TG2-TGF- $\beta$ /EMT gene signature in synovial cells and tissues from RA patients. Accordingly, TGF- $\beta$ and TG2 stimulated Snail-induced invadosome production in rat and human synovial cells. More important, down-regulation of Snail in CIA joints prevented invasion of the articular cartilage by the hyperplastic synovium and cartilage degradation. Our findings not only reveal, for the first time, 
A

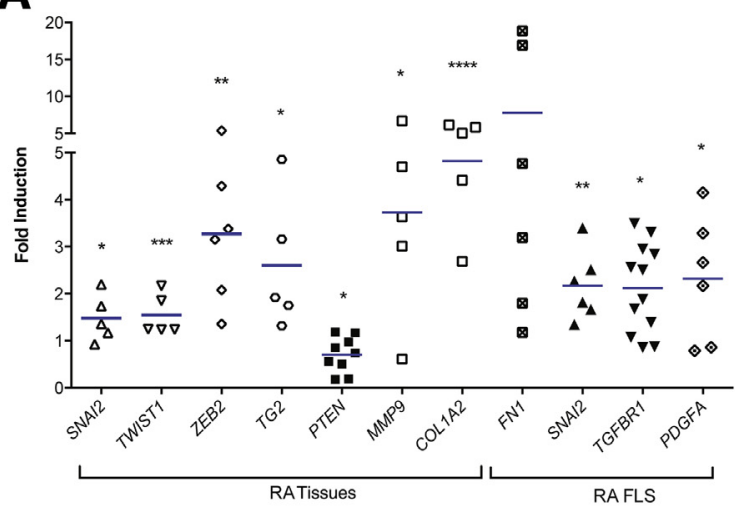

C

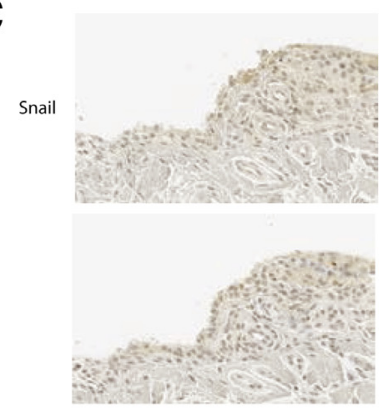

$\mathrm{OA} 1413$

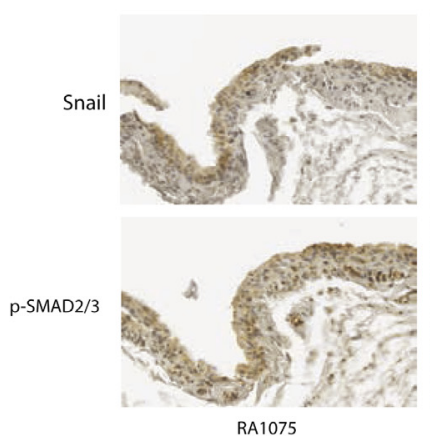

B

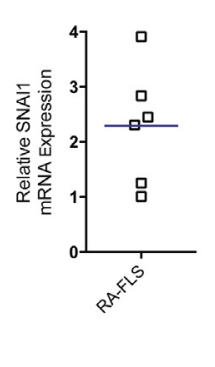

D

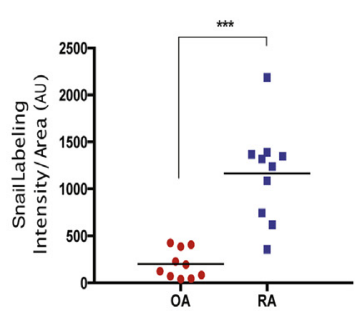

Figure 5 Snail is overexpressed in human rheumatoid arthritis fibroblast-like synoviocytes (RA-FLS) and is essential for matrix degradation and invasion. A: Analysis of data extracted from public gene array databases [National Center for Biotechnology Information Gene Expression Omnibus (GEO); http://www.ncbi.nlm.nih.gov/geo] reveals increased expression of epithelialmesenchymal transition (EMT)-related genes in synovial tissues and/or cultured synoviocytes of RA patients. Primary data were derived from data sets under the GEO accession numbers GSE7307 (ZEB2 and FN1), GSE1919 (SNAI2 in synovial tissues, TWIST1, TG2, MMP9, COL1A2, and PTEN), GSE7669 (SNAI2 in cultured FLS, TGFBR1, and PDGFA), and GSE2053 (PTEN). Results are shown as fold increases in RA gene expression relative to control [osteoarthritis (OA) for GSE1919 and GSE7669; normal tissue for GSE7307]. B: RT-qPCR analysis of Snai1 mRNA expression using Rplp0 as a housekeeping gene. Results are expressed as mean mRNA levels in RA-FLS relative to control (C)-FLS. C: Representative images of Snail, pSmad2/3 immunostaining, and control rabbit IgG of synovial tissues of OA and RA patients. D: The associated graph shows relative labeling intensities in arbitrary units (AUs) for $10 \mathrm{OA}$ and 10 RA patients. Data represent means \pm SEM (unpaired $t$-test; A, B, and D). $n=6$ (B, RA-FLS); $n=4$ (B, C-FLS). ${ }^{*} P<0.05,{ }^{*} P<<0.01$, $* * * P<0.001$, and $* * * * P<0.0001$. Original magnification, $\times 40$ (C).

how Snail contributes to the prodestructive properties of FLS, but also provide a rationale to subvert cartilage erosion by synoviocytes using Snail-targeted therapy.

Snail is a transcription factor implicated in a broad spectrum of biological functions, including regulation of cell proliferation, differentiation, invasion, and survival in a variety of cells. ${ }^{21,22,24,27}$ In a recent study, Snail overexpression was shown to increase cytokine expression and invasiveness of rat synovial cells, resulting in the exacerbation of CIA. ${ }^{35}$ In this study, we highlight the direct implication of Snaill that acts as a key effector of ECM degradation in arthritic FLS by promoting invadosome formation. Snail was specifically associated with the FLS subpopulation responsible for active matrix degradation and overexpressed in synovial membranes of CIA joints. Accordingly, Snail-deficient A-FLS lose their ability to form ECM-degrading structures, whereas Snail knockdown in joints prevents cartilage invasion and degradation. Consistent with these findings, gene ontology analysis of Snail knockdown fibroblasts has demonstrated that Snail can orchestrate multiple processes, which are essential to fibroblast motile behavior, including adhesion, migration, and proteolysis. In particular, cortactin, RhoA, ROCK1, and MT1-MMP, important molecules in the biology of invadopodia, were found to be regulated in Snail-depleted cells. ${ }^{33}$ Snail has also been shown to up-regulate the expression and activity of MMP-2, MMP-3, MMP-9, and MT1-MMP in different carcinoma cell lines. ${ }^{13,14,61}$ Even though Snail overexpression or Snail depletion failed to regulate MT1MMP expression in FLS (Supplemental Figure S5), high levels of Snai1 expression in these cells paralleled increases in metalloproteinase expression (MMP-9 and MMP-3) (Supplemental Table S1). MMP-9 was previously found to be enriched at the invadopodia of cancer cells, ${ }^{62,63}$ and MMP-3 was associated with ECM degradation at sites of invadosome formation in A-FLS. ${ }^{8}$ These findings suggest that during the course of arthritis, synoviocytes reactivate the expression of the transcription factor Snail to gain their 


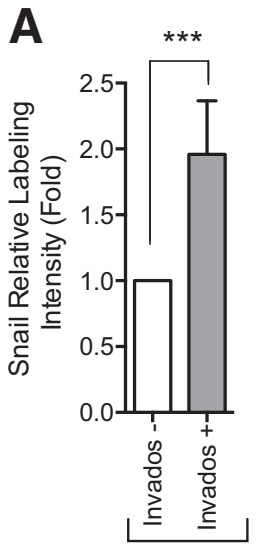

RA-FLS
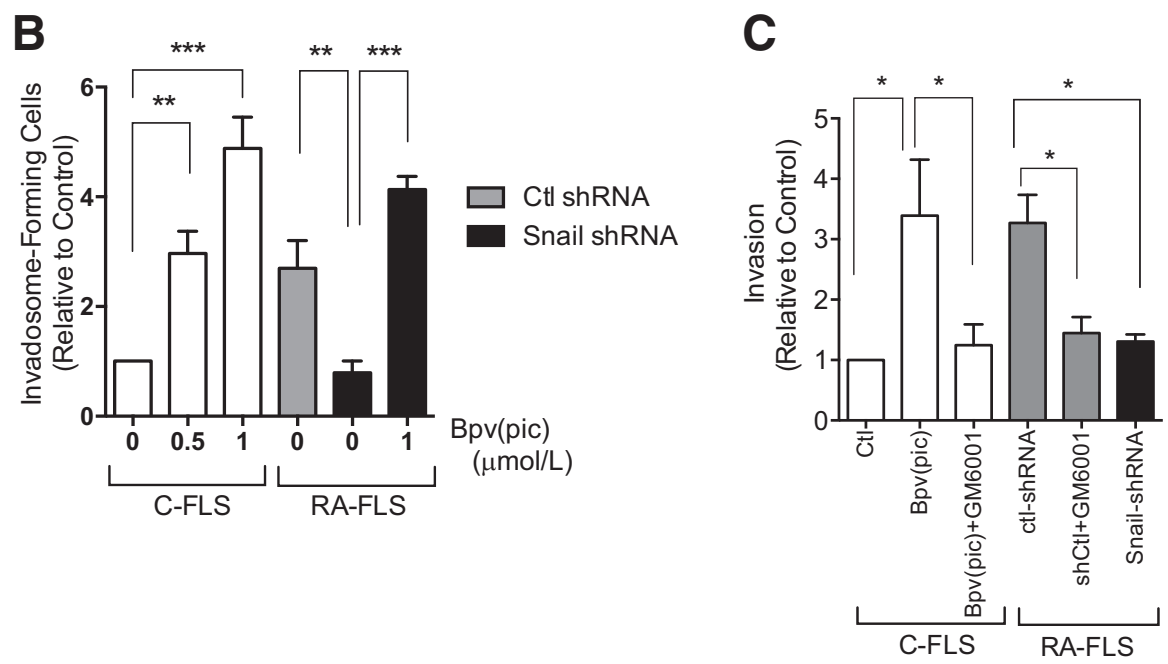

Figure 6 Snail is essential for matrix degradation and invasion by human rheumatoid arthritis fibroblast-like synoviocytes (RA-FLS). A: RA-FLS were grown on $1 \%$ Alexa Fluor 488-labeled gelatin (green) and immunostained for Snail. Intensity was measured for cells associated [invadosome (invado + )] or not (invado - ) with areas of matrix degradation. B: Control (C)-FLS or RA-FLS transduced with Snail-shRNA or control (Ctl)-shRNA treated with bpv(pic) for 40 hours in invadosome assay. C: Similar experiments performed for 72 hours in collagen type II-coated transwell invasion assay. For this assay, cells were also incubated in the presence or absence of $10 \mu \mathrm{g} / \mathrm{mL}$ GM6001. Results are expressed as means + SEM (A, paired $t$-test, intensity relative to cells not forming invadopodia for 100 cells; B, analysis of variance; and $\mathbf{C}$, unpaired $t$-test, relative to control cells). $n=3(\mathbf{A}) ; n=4$ (B and $\mathbf{C}) .{ }^{*} P<0.05,{ }^{* *} P<0.01$, and ${ }^{* * *} P<0.001$.

invasive and prodestructive phenotype through the formation of protease-enriched degrading devices, the invadosomes.

\section{The TG2/TGF- $\beta$ Axis as a Trigger for Snail Expression}

Snail expression in FLS of CIA rats and RA patients was associated with an EMT gene signature characteristic of TGF- $\beta /$ TG2 signaling. TGF- $\beta$ is a major inducer of EMT in carcinogenesis. ${ }^{64}$ Moreover, we have previously shown that endogenous TGF- $\beta$ production and signaling, triggered by TG2 activity, play a critical role in the progression of experimental arthritis by promoting invadosome-induced cartilage breakdown. ${ }^{10} \mathrm{We}$, therefore, hypothesized that the presence of TG2 and/or TGF- $\beta$ in synovial lining would serve as potent stimuli to increase FLS aggressiveness by controlling cues involved in the EMT program. We show herein that both TG2 and TGF- $\beta$ increase Snail expression in FLS and that TG2-dependent induction of Snail is partly dependent on TGF- $\beta$ signaling. Furthermore, knockdown of Snail markedly reduced the ability of A-FLS to produce invadosomes in response to TGF- $\beta$ or TG2. Our findings point to the TG2/TGF- $\beta$ axis as a potential trigger for Snail expression in synovial joints. However, we cannot rule out the participation of other potent EMT inducers, such as PDGF, which has been shown to be overexpressed in joints. In addition, tumor necrosis factor was recently shown to up-regulate Snail expression in synovial cells. ${ }^{35}$ The ability of PDGF-B and TGF- $\beta$ to strongly potentiate the response of FLS to cytokines, such as tumor necrosis factor or IL-1, suggests that Snail overexpression in joints might rely on a complex network of cytokines and growth factors that include TGF- $\beta$. $^{65}$

\section{Mechanisms of Snail-Induced Invadosome Formation Involve the PTEN-PDGFR/PI3K Axis}

Few direct targets of Snail have been identified. Among them, Snail has been shown to bind the PTEN promoter in cancer cells, ${ }^{24}$ repressing its activity. Herein, we found that Snail down-regulates the expression of PTEN in synoviocytes and that PTEN expression is reduced in synoviocytes derived from arthritic joints compared with control synoviocytes. Our findings confirm prior studies that showed that PTEN is not detected in the lining layer of RA synovium compared with normal tissue or in RA synoviocytes invading human cartilage in a severe combined immunodeficiency mouse coimplantation model. ${ }^{48} \mathrm{We}$ also observed that PTEN inhibition in arthritic FLS reversed the loss of invadoposome formation caused by Snail depletion, indicating that Snail induces ECM degradation through PTEN repression. Therefore, high expression of Snail with concomitant low expression of PTEN seems to be a discriminative feature of the prodestructive phenotype of FLS in arthritis.

PTEN is known to facilitate cell migration and invasion through its action on multiple targets. Most of the PTEN suppressor functions have been attributed to its lipid phosphatase activity that antagonizes the action of PI3K by converting phosphatidylinositol-3,4,5-triphosphate (PIP3) to phosphatidylinositol-4,5-bisphosphate (PIP2). This action restricts the recruitment and activation of pleckstrin homology domain-harboring PI3K effector proteins, such as AKT and PDK1. ${ }^{66}$ Accordingly, our data indicated that PTEN inhibition in arthritic FLS results in a strong increase in AKT phosphorylation and that both PI3K and AKT activities were necessary for invadosome-associated ECM degradation. The formation of invadopodia in cancer cells or 


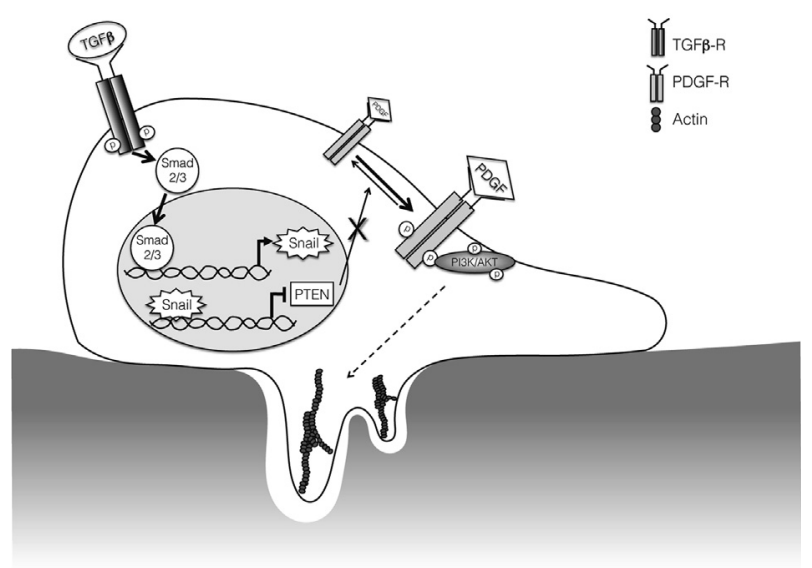

Figure 7 Schematic model of the role of Snail in invadosome formation by arthritic synovial cells. Work presented herein indicates that Snail, up-regulated in arthritic synoviocytes by the transglutaminase 2 (TG2)/ transforming growth factor (TGF)- $\beta /$ Smad axis, directly suppresses PTEN transcription. Down-regulation of PTEN leads to an increase in plateletderived growth factor receptor (PDGFR) phosphorylation/activation that induces the formation of invadosomes through phosphatidylinositol 3-kinase/AKT activation. Promising targets for chemical inhibition include the TG2/TGF- $\beta$ axis, Snail, and PDGFR signaling. PTEN, phosphatase and tensin homolog; TGF- $\beta R$, TGF- $\beta$ receptor.

podosomes in osteoclasts has also been reported to require PI3K activity or PIP3 availability. ${ }^{67-69}$ Although the molecular targets of the PI3K pathway in cell invasion have not been clearly identified, inhibition of this pathway was found to modulate MMP production and PDGF-induced F-actin rearrangement, ${ }^{70}$ a process involved in podosome and invadopodia formation. ${ }^{71,72}$ In addition, PIP3 may also serve as a precursor of PIP2, which has been shown to regulate invadopodia formation by recruiting Tks5, a scaffolding protein essential for F-actin core formation. ${ }^{73}$ It is, therefore, possible that PI3K inhibition suppresses invadosome-driven ECM degradation by impeding both invadosome core formation and MMP-mediated proteolysis.

Another emerging target of PTEN is the receptor tyrosine kinase (RTK) PDGFR. Through its protein phosphatase activity, PTEN has been shown to bind and dephosphorylate the activated PDGFR in mesangial cells, impeding downstream PI3K/AKT signaling. ${ }^{55}$ PDGFR family RTK, namely PDGFR $\alpha$ and PDGFR $\beta$, and their ligands, PDGF A to D, have previously been implicated in RA-FLS aggressiveness. Increased levels of the PDGF receptors and ligands were detected in the synovial lining and in FLS cultures of RA patients. ${ }^{52,74,75}$ Furthermore, PDGF has been found to regulate synoviocyte proliferation, anchorage-independent growth, and collagenase transcription, which are features known to be part of the transformed and prodestructive phenotype of RA synoviocytes. ${ }^{53,54,76}$ However, the impact of PTEN regulation in synoviocytes on PDGFR activation and ensuing biological functions has not yet been demonstrated. We report herein that inhibition of PTEN activity in arthritic FLS resulted in increased phosphorylation of PDGFR that was required for the invadosome-driven ECM degradation capabilities of the cells. Enhanced activation of PDGFR was associated with a corresponding increase in AKT phosphorylation, an event that was partly dependent on PDGFR kinase activity. These findings suggest that invadosome formation in A-FLS depends on an autocrine RTK/ PI3K/AKT activation loop that involves repression of the PTEN phosphatase. Although the involvement of other RTKs and phosphatases ${ }^{77}$ cannot be excluded, our studies suggest that PTEN is the primary PDGFR phosphatase causally involved in PTEN-driven PDGFR phosphorylation. Aberrant activation of RTKs has been linked to malignant progression in many human cancers, ${ }^{78}$ and various studies have reported an up-regulation of tyrosine kinase activity in RA synoviocytes. $^{79,80}$ Furthermore, the balance between RTK and phosphatase activities is an emerging mechanism that influences cancer progression and resistance to treatment. ${ }^{81}$ It is, therefore, likely that the reduction of PTEN expression observed in Snail-expressing cells within the RA synovium contributes to an imbalance of tyrosine kinases and phosphatases, hence promoting the tumor-like phenotype of synovial cells that involves enhanced invadosome production, cell invasion, and cartilage destruction.

\section{Components of the EMT Program as Potential Therapeutic Targets or Markers in RA}

Despite impressive developments in RA therapeutics during the past 20 years, results of clinical trials show that the various treatment strategies have only a moderate effect on joint destruction, ${ }^{82-85}$ although encouraging results have been reported using a combination of methotrexate with novel biological treatments, such as tumor necrosis factor inhibitors in subsets of patients. ${ }^{86}$ However, these biological agents are associated with several adverse effects and concerns, such as increased susceptibility to infection, potential risks of malignancies, and lack of response in a proportion of patients. Herein, we showed that cartilage invasion and degradation in CIA was efficiently prevented by blockade of Snail. Because beyond embryonic development, EMT components are only reactivated under stress or pathological conditions, such dependency on Snail or its associated gene expression program for synovial cell-driven joint damage could possibly be exploited to improve diagnosis and treatment of RA. In agreement with this possibility, recent data from cancer patients have indicated that reversal of EMT by apricoxib, which blocks downstream TGF$\beta$-induced EMT, was associated with inhibition of cancer progression in several types of tumors. ${ }^{87-89}$ Similarly, retrospective analysis of tumor samples from clinical trials of erlotinib (an epidermal growth factor receptor antagonist) revealed an association between E-cadherin and vimentin expression and positive response to erlotinib in patients with refractory lung carcinoma. ${ }^{90}$ These data indicate that EMT inducers or components of the EMT program are potential therapeutic targets, as well as predictive markers of response to treatment. Future investigation will be needed to evaluate 
the expression pattern and clinical outcome of Snail and its associated gene expression program in RA patients.

In conclusion, our study provides direct evidence that Snail is a central component of the prodestructive, invadosome-forming phenotype of synoviocytes and identify a novel Snail-PTEN-PDGFR/PI3K pathway by which Snail exerts its effector function. Although our data suggest a link between components of the EMT program cartilage invasion and joint damage, further work is required to elucidate how these pathological changes evolve during the establishment and progression of the disease and whether they can be of clinical relevance in the stratification and/or treatment of patients experiencing rapid joint degradation and candidates in need of aggressive and timely treatment.

\section{Acknowledgments}

We thank Dr. Gilles Dupuis for critical reading of the manuscript; Dr. Leonid Volkov for assistance with confocal microscopy experiments; Dr. Gail V.W. Johnson (University of Rochester, Rochester, NY) for generously providing pcDNA3.1; Bob Weinberg for providing pTK-Snail (addgene plasmid 36976); and Joshua Mendell for providing pGL3wtPTEN (addgene plasmid 21326).

\section{Supplemental Data}

Supplemental material for this article can be found at http://dx.doi.org/10.1016/j.ajpath.2015.10.021.

\section{References}

1. Meinecke I, Rutkauskaite E, Gay S, Pap T: The role of synovial fibroblasts in mediating joint destruction in rheumatoid arthritis. Curr Pharm Des 2005, 11:563-568

2. Muller-Ladner U, Kriegsmann J, Franklin BN, Matsumoto S, Geiler T, Gay RE, Gay S: Synovial fibroblasts of patients with rheumatoid arthritis attach to and invade normal human cartilage when engrafted into SCID mice. Am J Pathol 1996, 149: $1607-1615$

3. Firestein GS: Invasive fibroblast-like synoviocytes in rheumatoid arthritis. Passive responders or transformed aggressors? Arthritis Rheum 1996, 39:1781-1790

4. Firestein GS, Paine MM: Stromelysin and tissue inhibitor of metalloproteinases gene expression in rheumatoid arthritis synovium. Am J Pathol 1992, 140:1309-1314

5. Pap T, Muller-Ladner U, Gay RE, Gay S: Fibroblast biology: role of synovial fibroblasts in the pathogenesis of rheumatoid arthritis. Arthritis Res 2000, 2:361-367

6. Tolboom TC, van der Helm-Van Mil AH, Nelissen RG, Breedveld FC, Toes RE, Huizinga TW: Invasiveness of fibroblastlike synoviocytes is an individual patient characteristic associated with the rate of joint destruction in patients with rheumatoid arthritis. Arthritis Rheum 2005, 52:1999-2002

7. Bartok B, Firestein GS: Fibroblast-like synoviocytes: key effector cells in rheumatoid arthritis. Immunol Rev 2010, 233:233-255

8. Lauzier A, Charbonneau M, Harper K, Jilaveanu-Pelmus M, Dubois CM: Formation of invadopodia-like structures by synovial cells promotes cartilage breakdown in collagen-induced arthritis involvement of the protein tyrosine kinase Src. Arthritis Rheum 2011 63:1591-1602

9. Linder S: Invadosomes at a glance. J Cell Sci 2009, 122:3009-3013

10. Lauzier A, Charbonneau M, Paquette M, Harper K, Dubois CM: Transglutaminase 2 cross-linking activity is linked to invadopodia formation and cartilage breakdown in arthritis. Arthritis Res Ther 2012, 14:R159

11. Thiery JP, Acloque H, Huang RY, Nieto MA: Epithelialmesenchymal transitions in development and disease. Cell 2009, 139:871-890

12. Yilmaz M, Christofori G: EMT, the cytoskeleton, and cancer cell invasion. Cancer Metastasis Rev 2009, 28:15-33

13. Sun L, Diamond ME, Ottaviano AJ, Joseph MJ, Ananthanarayan V, Munshi HG: Transforming growth factor-beta 1 promotes matrix metalloproteinase-9-mediated oral cancer invasion through snail expression. Mol Cancer Res 2008, 6:10-20

14. Yokoyama K, Kamata N, Fujimoto R, Tsutsumi S, Tomonari M, Taki M, Hosokawa H, Nagayama M: Increased invasion and matrix metalloproteinase-2 expression by Snail-induced mesenchymal transition in squamous cell carcinomas. Int J Oncol 2003, 22:891-898

15. Taketazu F, Kato M, Gobl A, Ichijo H, ten Dijke P, Itoh J, et al: Enhanced expression of transforming growth factor-beta $\mathrm{S}$ and transforming growth factor-beta type II receptor in the synovial tissues of patients with rheumatoid arthritis. Lab Invest 1994, 70: 620-630

16. Fava R, Olsen N, Keski-Oja J, Moses H, Pincus T: Active and latent forms of transforming growth factor beta activity in synovial effusions. J Exp Med 1989, 169:291-296

17. Manabe N, Oda H, Nakamura K, Kuga Y, Uchida S, Kawaguchi H: Involvement of fibroblast growth factor-2 in joint destruction of rheumatoid arthritis patients. Rheumatology (Oxford) 1999, 38: 714-720

18. Thomas JW, Thieu TH, Byrd VM, Miller GG: Acidic fibroblast growth factor in synovial cells. Arthritis Rheum 2000, 43: 2152-2159

19. Bowden ET, Onikoyi E, Slack R, Myoui A, Yoneda T, Yamada KM, Mueller SC: Co-localization of cortactin and phosphotyrosine identifies active invadopodia in human breast cancer cells. Exp Cell Res 2006, 312:1240-1253

20. Frame MC: Newest findings on the oldest oncogene; how activated src does it. J Cell Sci 2004, 117:989-998

21. Vega S, Morales AV, Ocana OH, Valdes F, Fabregat I, Nieto MA: Snail blocks the cell cycle and confers resistance to cell death. Genes Dev 2004, 18:1131-1143

22. Kajita M, McClinic KN, Wade PA: Aberrant expression of the transcription factors snail and slug alters the response to genotoxic stress. Mol Cell Biol 2004, 24:7559-7566

23. Tribulo C, Aybar MJ, Sanchez SS, Mayor R: A balance between the anti-apoptotic activity of Slug and the apoptotic activity of msx 1 is required for the proper development of the neural crest. Dev Biol 2004, 275:325-342

24. Escriva M, Peiro S, Herranz N, Villagrasa P, Dave N, MontserratSentis B, Murray SA, Franci C, Gridley T, Virtanen I, Garcia de Herreros A: Repression of PTEN phosphatase by Snaill transcriptional factor during gamma radiation-induced apoptosis. Mol Cell Biol 2008, 28:1528-1540

25. Kurrey NK, Jalgaonkar SP, Joglekar AV, Ghanate AD, Chaskar PD, Doiphode RY, Bapat SA: Snail and slug mediate radioresistance and chemoresistance by antagonizing p53-mediated apoptosis and acquiring a stem-like phenotype in ovarian cancer cells. Stem Cells 2009, 27:2059-2068

26. Southall TD, Brand AH: Neural stem cell transcriptional networks highlight genes essential for nervous system development. EMBO J 2009, 28:3799-3807

27. Barrallo-Gimeno A, Nieto MA: The Snail genes as inducers of cell movement and survival: implications in development and cancer. Development 2005, 132:3151-3161 
28. Fang R, Zhang G, Guo Q, Ning F, Wang H, Cai S, Du J: Nodal promotes aggressive phenotype via Snail-mediated epithelial-mesenchymal transition in murine melanoma. Cancer Lett 2013, 333:66-75

29. Kim JJ, Yin B, Christudass CS, Terada N, Rajagopalan K, Fabry B, Lee DY, Shiraishi T, Getzenberg RH, Veltri RW, An SS, Mooney SM: Acquisition of paclitaxel resistance is associated with a more aggressive and invasive phenotype in prostate cancer. J Cell Biochem 2013, 114:1286-1293

30. Kashyap A, Zimmerman T, Ergul N, Bosserhoff A, Hartman U, Alla V, Bataille F, Galle PR, Strand S, Strand D: The human Lgl polarity gene, Hugl-2, induces MET and suppresses Snail tumorigenesis. Oncogene 2013, 32:1396-1407

31. Liu S, Kumar SM, Martin JS, Yang R, Xu X: Snaill mediates hypoxia-induced melanoma progression. Am J Pathol 2011, 179: 3020-3031

32. Franci C, Takkunen M, Dave N, Alameda F, Gomez S, Rodriguez R, Escriva M, Montserrat-Sentis B, Baro T, Garrido M, Bonilla F, Virtanen I, Garcia de Herreros A: Expression of Snail protein in tumor-stroma interface. Oncogene 2006, 25:5134-5144

33. Rowe RG, Li XY, Hu Y, Saunders TL, Virtanen I, Garcia de Herreros A, Becker KF, Ingvarsen S, Engelholm LH, Bommer GT, Fearon ER, Weiss SJ: Mesenchymal cells reactivate Snail1 expression to drive three-dimensional invasion programs. J Cell Biol 2009, 184: 399-408

34. Hotz B, Visekruna A, Buhr HJ, Hotz HG: Beyond epithelial to mesenchymal transition: a novel role for the transcription factor Snail in inflammation and wound healing. J Gastrointest Surg 2010, 14: 388-397

35. Chen SY, Shiau AL, Li YT, Lin CC, Jou IM, Liu MF, Wu CL, Wang CR: Transcription factor snail regulates tumor necrosis factor alpha-mediated synovial fibroblast activation in the rheumatoid joint. Arthritis Rheumatol 2015, 67:39-50

36. Pine PR, Chang B, Schoettler N, Banquerigo ML, Wang S, Lau A, Zhao F, Grossbard EB, Payan DG, Brahn E: Inflammation and bone erosion are suppressed in models of rheumatoid arthritis following treatment with a novel Syk inhibitor. Clin Immunol 2007, 124: 244-257

37. Blanchette F, Day R, Dong W, Laprise MH, Dubois CM: TGFbeta1 regulates gene expression of its own converting enzyme furin. J Clin Invest 1997, 99:1974-1983

38. Guo W, Keckesova Z, Donaher JL, Shibue T, Tischler V, Reinhardt F, Itzkovitz S, Noske A, Zurrer-Hardi U, Bell G, Tam WL, Mani SA, van Oudenaarden A, Weinberg RA: Slug and Sox9 cooperatively determine the mammary stem cell state. Cell 2012, 148: $1015-1028$

39. O'Donnell KA, Wentzel EA, Zeller KI, Dang CV, Mendell JT: c-Myc-regulated microRNAs modulate E2F1 expression. Nature 2005, 435:839-843

40. Charbonneau M, Harper K, Grondin F, Pelmus M, McDonald PP, Dubois CM: Hypoxia-inducible factor mediates hypoxic and tumor necrosis factor alpha-induced increases in tumor necrosis factor-alpha converting enzyme/ADAM17 expression by synovial cells. J Biol Chem 2007, 282:33714-33724

41. Blanchette F, Rivard N, Rudd P, Grondin F, Attisano L, Dubois CM: Cross-talk between the p42/p44 MAP kinase and Smad pathways in transforming growth factor beta 1-induced furin gene transactivation. J Biol Chem 2001, 276:33986-33994

42. Aletaha D, Neogi T, Silman AJ, Funovits J, Felson DT, Bingham CO 3rd, Birnbaum NS, Burmester GR, Bykerk VP, Cohen MD, Combe B, Costenbader KH, Dougados M, Emery P, Ferraccioli G, Hazes JM, Hobbs K, Huizinga TW, Kavanaugh A, Kay J, Kvien TK, Laing T, Mease P, Menard HA, Moreland LW, Naden RL, Pincus T, Smolen JS, Stanislawska-Biernat E, Symmons D, Tak PP, Upchurch KS, Vencovsky J, Wolfe F, Hawker G: 2010 Rheumatoid arthritis classification criteria: an American College of Rheumatology/European League Against
Rheumatism collaborative initiative. Arthritis Rheum 2010, 62: $2569-2581$

43. Ranganathan P, Agrawal A, Bhushan R, Chavalmane AK, Kalathur RK, Takahashi T, Kondaiah P: Expression profiling of genes regulated by TGF-beta: differential regulation in normal and tumour cells. BMC Genomics 2007, 8:98

44. Muzio M, Sironi M, Polentarutti N, Mantovani A, Colotta F: Induction by transforming growth factor-beta 1 of the interleukin-1 receptor antagonist and of its intracellular form in human polymorphonuclear cells. Eur J Immunol 1994, 24:3194-3198

45. Remst DF, Blom AB, Vitters EL, Bank RA, van den Berg WB, Blaney Davidson EN, van der Kraan PM: Gene expression analysis of osteoarthritis synovium reveals elevation of transforming growth factor-beta responsive genes in osteoarthritis-related fibrosis. Arthritis Rheum 2014, 66:647-656

46. Inagaki Y, Truter S, Ramirez F: Transforming growth factor-beta stimulates alpha 2(I) collagen gene expression through a cis-acting element that contains an Sp1-binding site. J Biol Chem 1994, 269: $14828-14834$

47. Kannan K, Ortmann RA, Kimpel D: Animal models of rheumatoid arthritis and their relevance to human disease. Pathophysiology 2005, 12:167-181

48. Pap T, Franz JK, Hummel KM, Jeisy E, Gay R, Gay S: Activation of synovial fibroblasts in rheumatoid arthritis: lack of Expression of the tumour suppressor PTEN at sites of invasive growth and destruction. Arthritis Res 2000, 2:59-64

49. Lai JP, Dalton JT, Knoell DL: Phosphatase and tensin homologue deleted on chromosome ten (PTEN) as a molecular target in lung epithelial wound repair. Br J Pharmacol 2007, 152:1172-1184

50. Schmid AC, Byrne RD, Vilar R, Woscholski R: Bisperoxovanadium compounds are potent PTEN inhibitors. FEBS Lett 2004, 566: $35-38$

51. Artym VV, Zhang Y, Seillier-Moiseiwitsch F, Yamada KM, Mueller SC: Dynamic interactions of cortactin and membrane type 1 matrix metalloproteinase at invadopodia: defining the stages of invadopodia formation and function. Cancer Res 2006, 66:3034-3043

52. Pohlers D, Huber R, Ukena B, Kinne RW: Expression of plateletderived growth factors $\mathrm{C}$ and $\mathrm{D}$ in the synovial membrane of patients with rheumatoid arthritis and osteoarthritis. Arthritis Rheum 2006, 54:788-794

53. Butler DM, Leizer T, Hamilton JA: Stimulation of human synovial fibroblast DNA synthesis by platelet-derived growth factor and fibroblast growth factor: differences to the activation by IL-1. J Immunol 1989, 142:3098-3103

54. Lafyatis R, Remmers EF, Roberts AB, Yocum DE, Sporn MB, Wilder RL: Anchorage-independent growth of synoviocytes from arthritic and normal joints: stimulation by exogenous platelet-derived growth factor and inhibition by transforming growth factor-beta and retinoids. J Clin Invest 1989, 83:1267-1276

55. Mahimainathan L, Choudhury GG: Inactivation of platelet-derived growth factor receptor by the tumor suppressor PTEN provides a novel mechanism of action of the phosphatase. J Biol Chem 2004, 279:15258-15268

56. Valius M, Kazlauskas A: Phospholipase C-gamma 1 and phosphatidylinositol 3 kinase are the downstream mediators of the PDGF receptor's mitogenic signal. Cell 1993, 73:321-334

57. Furuta T, Sakai T, Senga T, Osawa T, Kubo K, Shimizu T, Suzuki R, Yoshino T, Endo M, Miwa A: Identification of potent and selective inhibitors of PDGF receptor autophosphorylation. J Med Chem 2006, 49:2186-2192

58. Chou YT, Wang H, Chen Y, Danielpour D, Yang YC: Cited2 modulates TGF-beta-mediated upregulation of MMP9. Oncogene 2006, 25:5547-5560

59. Ebert MP, Fei G, Schandl L, Mawrin C, Dietzmann K, Herrera P, Friess H, Gress TM, Malfertheiner P: Reduced PTEN expression in the pancreas overexpressing transforming growth factor-beta 1 . Br J Cancer 2002, 86:257-262 
60. Chow JY, Ban M, Wu HL, Nguyen F, Huang M, Chung H, Dong H, Carethers JM: TGF-beta downregulates PTEN via activation of NF-kappaB in pancreatic cancer cells. Am J Physiol Gastrointest Liver Physiol 2010, 298:G275-G282

61. Jiang W, Zhang Y, Kane KT, Collins MA, Simeone DM, di Magliano MP, Nguyen KT: CD44 regulates pancreatic cancer invasion through MT1-MMP. Mol Cancer Res 2015, 13:9-15

62. Murphy DA, Courtneidge SA: The "ins" and "outs" of podosomes and invadopodia: characteristics, formation and function. Nat Rev Mol Cell Biol 2011, 12:413-426

63. Jacob A, Jing J, Lee J, Schedin P, Gilbert SM, Peden AA, Junutula JR, Prekeris R: Rab40b regulates trafficking of MMP2 and MMP9 during invadopodia formation and invasion of breast cancer cells. J Cell Sci 2013, 126:4647-4658

64. Giannelli G, Villa E, Lahn M: Transforming growth factor-beta as a therapeutic target in hepatocellular carcinoma. Cancer Res 2014, 74: 1890-1894

65. Rosengren S, Corr M, Boyle DL: Platelet-derived growth factor and transforming growth factor beta synergistically potentiate inflammatory mediator synthesis by fibroblast-like synoviocytes. Arthritis Res Ther 2010, 12:R65

66. Song MS, Salmena L, Pandolfi PP: The functions and regulation of the PTEN tumour suppressor. Nat Rev Mol Cell Biol 2012, 13: 283-296

67. Hoshino D, Jourquin J, Emmons SW, Miller T, Goldgof M, Costello K, Tyson DR, Brown B, Lu Y, Prasad NK, Zhang B, Mills GB, Yarbrough WG, Quaranta V, Seiki M, Weaver AM: Network analysis of the focal adhesion to invadopodia transition identifies a PI3K-PKCalpha invasive signaling axis. Sci Signal 2012, 5:ra66

68. Yamaguchi H, Yoshida S, Muroi E, Yoshida N, Kawamura M, Kouchi Z, Nakamura Y, Sakai R, Fukami K: Phosphoinositide 3-kinase signaling pathway mediated by p110alpha regulates invadopodia formation. J Cell Biol 2011, 193:1275-1288

69. Chellaiah MA, Biswas RS, Yuen D, Alvarez UM, Hruska KA: Phosphatidylinositol 3,4,5-trisphosphate directs association of Src homology 2-containing signaling proteins with gelsolin. J Biol Chem 2001, 276:47434-47444

70. Brachmann SM, Yballe CM, Innocenti M, Deane JA, Fruman DA, Thomas SM, Cantley LC: Role of phosphoinositide 3-kinase regulatory isoforms in development and actin rearrangement. Mol Cell Biol 2005, 25:2593-2606

71. Burgstaller G, Gimona M: Actin cytoskeleton remodelling via local inhibition of contractility at discrete microdomains. J Cell Sci 2004, 117:223-231

72. Hoshino D, Branch KM, Weaver AM: Signaling inputs to invadopodia and podosomes. J Cell Sci 2013, 126:2979-2989

73. Yamaguchi H, Yoshida S, Muroi E, Kawamura M, Kouchi Z, Nakamura Y, Sakai R, Fukami K: Phosphatidylinositol 4,5-bisphosphate and PIP5-kinase Ialpha are required for invadopodia formation in human breast cancer cells. Cancer Sci 2010, 101:1632-1638

74. Watanabe N, Ando K, Yoshida S, Inuzuka S, Kobayashi M, Matsui N, Okamoto T: Gene expression profile analysis of rheumatoid synovial fibroblast cultures revealing the overexpression of genes responsible for tumor-like growth of rheumatoid synovium. Biochem Biophys Res Commun 2002, 294:1121-1129

75. Reuterdahl C, Tingstrom A, Terracio L, Funa K, Heldin CH, Rubin K: Characterization of platelet-derived growth factor betareceptor expressing cells in the vasculature of human rheumatoid synovium. Lab Invest 1991, 64:321-329
76. Kumkumian GK, Lafyatis R, Remmers EF, Case JP, Kim SJ, Wilder RL: Platelet-derived growth factor and IL-1 interactions in rheumatoid arthritis. Regulation of synoviocyte proliferation, prostaglandin production, and collagenase transcription. J Immunol 1989, $143: 833-837$

77. Haj FG, Verveer PJ, Squire A, Neel BG, Bastiaens PI: Imaging sites of receptor dephosphorylation by PTP1B on the surface of the endoplasmic reticulum. Science 2002, 295:1708-1711

78. Robertson SC, Tynan J, Donoghue DJ: RTK mutations and human syndromes: when good receptors turn bad. Trends Genet 2000, 16 : 368

79. Krausz S, Garcia S, Ambarus CA, de Launay D, Foster M, Naiman B, Iverson W, Connor JR, Sleeman MA, Coyle AJ, Hamann J, Baeten D, Tak PP, Reedquist KA: Angiopoietin-2 promotes inflammatory activation of human macrophages and is essential for murine experimental arthritis. Ann Rheum Dis 2012, 71:1402-1410

80. Yamane S, Ishida S, Hanamoto Y, Kumagai K, Masuda R, Tanaka K, Shiobara N, Yamane N, Mori T, Juji T, Fukui N, Itoh T, Ochi T, Suzuki R: Proinflammatory role of amphiregulin, an epidermal growth factor family member whose expression is augmented in rheumatoid arthritis patients. J Inflamm (Lond) 2008, 5:5

81. Sastry SK, Elferink LA: Checks and balances: interplay of RTKs and PTPs in cancer progression. Biochem Pharmacol 2011, 82:435-440

82. van der Heijde DM, van Riel PL, Nuver-Zwart IH, Gribnau FW, vad de Putte LB: Effects of hydroxychloroquine and sulphasalazine on progression of joint damage in rheumatoid arthritis. Lancet 1989, 1: 1036-1038

83. Wolfe F, Sharp JT: Radiographic outcome of recent-onset rheumatoid arthritis: a 19-year study of radiographic progression. Arthritis Rheum 1998, 41:1571-1582

84. van Aken J, Lard LR, le Cessie S, Hazes JM, Breedveld FC, Huizinga TW: Radiological outcome after four years of early versus delayed treatment strategy in patients with recent onset rheumatoid arthritis. Ann Rheum Dis 2004, 63:274-279

85. Smolen JS, Emery P: Efficacy and safety of leflunomide in active rheumatoid arthritis. Rheumatology (Oxford) 2000, 39(Suppl 1): $48-56$

86. Lipsky PE, van der Heijde DM, St Clair EW, Furst DE, Breedveld FC, Kalden JR, Smolen JS, Weisman M, Emery P, Feldmann M, Harriman GR, Maini RN: Anti-Tumor Necrosis Factor Trial in Rheumatoid Arthritis with Concomitant Therapy Study Group: Infliximab and methotrexate in the treatment of rheumatoid arthritis. N Engl J Med 2000, 343:1594-1602

87. Kirane A, Toombs JE, Larsen JE, Ostapoff KT, Meshaw KR, Zaknoen S, Brekken RA, Burrows FJ: Epithelial-mesenchymal transition increases tumor sensitivity to COX-2 inhibition by apricoxib. Carcinogenesis 2012, 33:1639-1646

88. Kirane A, Toombs JE, Ostapoff K, Carbon JG, Zaknoen S, Braunfeld J, Schwarz RE, Burrows FJ, Brekken RA: Apricoxib, a novel inhibitor of COX-2, markedly improves standard therapy response in molecularly defined models of pancreatic cancer. Clin Cancer Res 2012, 18:5031-5042

89. Rao PN, Grover RK: Apricoxib, a COX-2 inhibitor for the potential treatment of pain and cancer. IDrugs 2009, 12:711-722

90. Yauch RL, Januario T, Eberhard DA, Cavet G, Zhu W, Fu L, Pham TQ, Soriano R, Stinson J, Seshagiri S, Modrusan Z, Lin CY, O'Neill V, Amler LC: Epithelial versus mesenchymal phenotype determines in vitro sensitivity and predicts clinical activity of erlotinib in lung cancer patients. Clin Cancer Res 2005, 11: $8686-8698$ 